\title{
Facts and factors in the formation and stability of binary crystals.
}

Aneglo Gavezzotti, ${ }^{a}$ Valentina Colombo, ${ }^{a}$ and Leonardo Lo Presti ${ }^{* a, b, c}$

${ }^{a}$ Università degli Studi di Milano, Dipartimento di Chimica, Via Golgi 19, 20133 Milano, Italy

${ }^{\mathrm{b}}$ Center for Materials Crystallography, Aarhus University, Langelandsgade 140, 8000 Aarhus, Denmark

${ }^{\mathrm{c}}$ CNR-ISTM, Istituto di Scienze e Tecnologie Molecolari, Via Golgi 19 I-20133 Milano, Italy mail: leonardo.lopresti@,unimi.it

\section{SUPPORTING INFORMATION}




\section{Contents}

Resetting the geometry of $\mathrm{COOH}$ groups in aromatic carboxylic acids.................................... 3

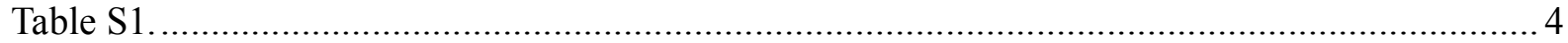

Table S2

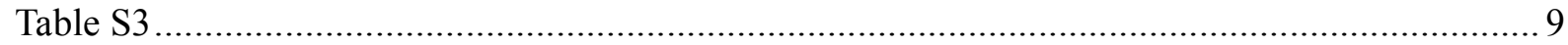

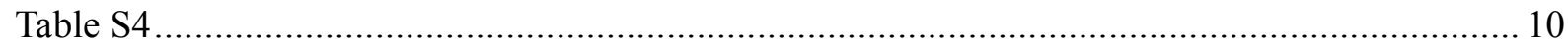

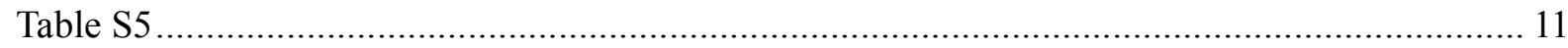

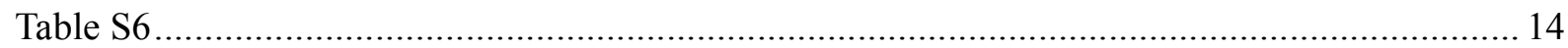

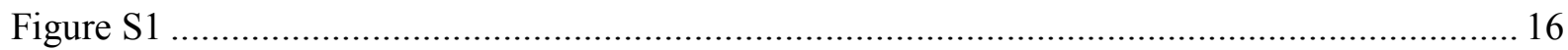

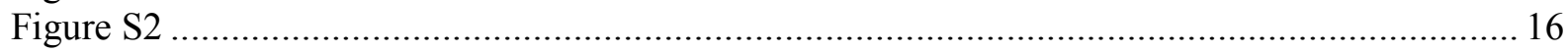

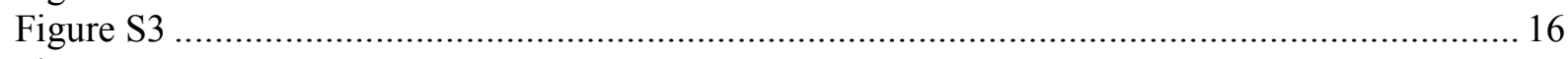

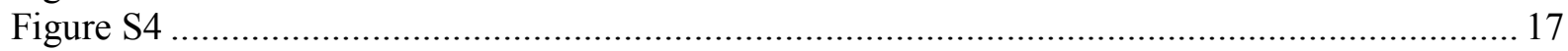

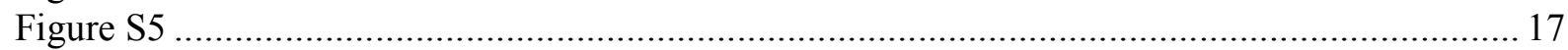

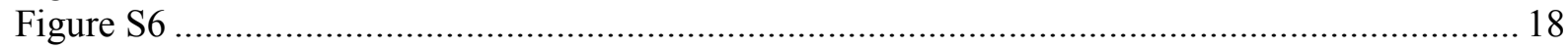

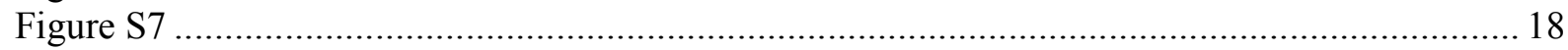

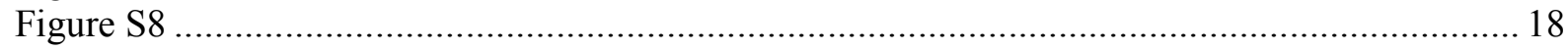

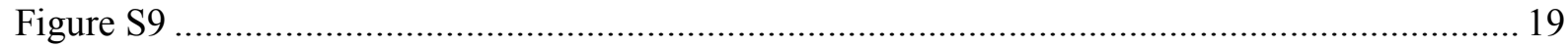

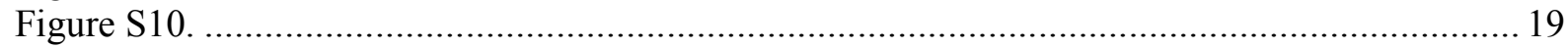

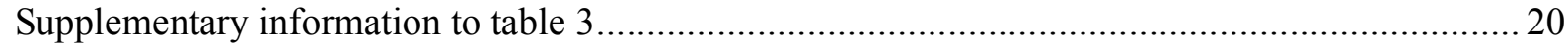

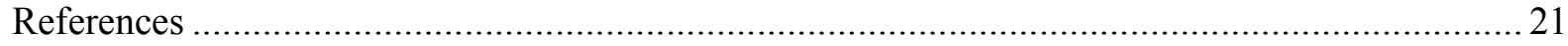




\section{Resetting the geometry of $\mathrm{COOH}$ groups in aromatic carboxylic acids.}

The carboxyl group attached to a phenyl ring, when a cyclic double $\mathrm{OH}^{\cdots} \mathrm{O}$ hydrogen bond is formed, almost invariably shows a disorder with single and double $\mathrm{C}-\mathrm{O}$ bond distances becoming more and more equal as the temperature is raised. The $\mathrm{D}(\mathrm{C}-\mathrm{O})$ difference goes from $0.1 \AA$ to zero and the proton positions are spread over two locations, or are not given at all. This disorder has been analysed. ${ }^{1}$ The disorder is temperature-dependent; therefore, it must be dynamic in nature. Ruling out an impossible flipping of the $\mathrm{COOH}$ group in the crystal, the most likely explanation is a dynamic shift of the proton along the $\mathrm{O} \cdots \mathrm{H}-\mathrm{O}$ line. One must assume that the flipping is instantaneous with a concomitant change of the two C-O bond lengths. Remarkably, this does not happen in aliphatic carboxylic acids.

For lattice energy calculations, one needs a reasonable model of the undistorted COOH group. A sample of 217 crystal structures of $\mathrm{Ph}-\mathrm{COOH}$ compounds was retrieved from the CSD with $\mathrm{T}(\mathrm{X}$-ray $)<100 \mathrm{~K}$. The peaks of the distributions of the geometrical parameters are illustrated in the following figure.

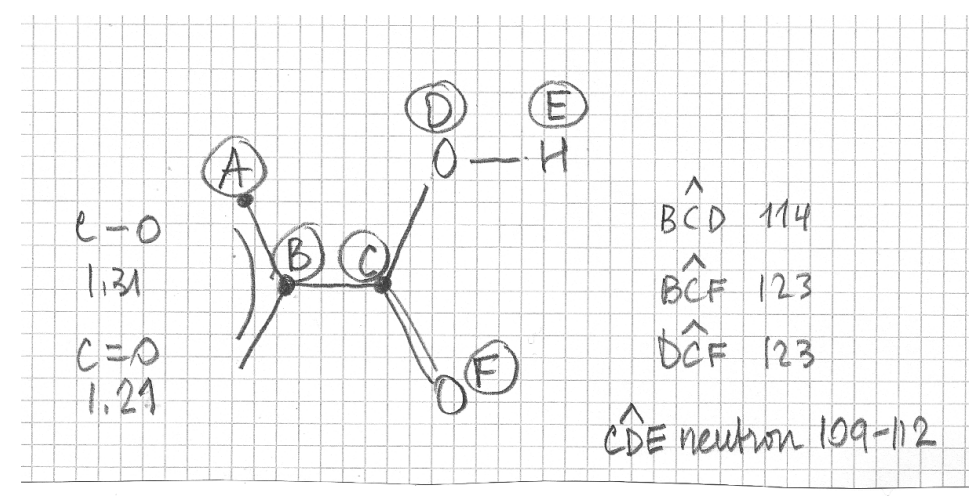

Although the $\mathrm{O}-\mathrm{H}$ bond length in X-rays is unreliable, the $\mathrm{COH}$ angle may be correctly approximated. The survey on the 217 structures yielded an average of $110^{\circ}$, while a survey of the few neutron determinations shows a range of 109$112^{\circ}$.

The experimental crystal structures were then corrected by:

1) using the experimental $\mathrm{ABCD}$ and $\mathrm{ABCF}$ torsion angles;

2) setting $\mathrm{C}=\mathrm{O} 1.21 \AA$ and $\mathrm{C}-\mathrm{O} 1.31 \AA$, with $\mathrm{O}-\mathrm{H} 1.00 \AA$ this last value being one of the calibration parameters in the PIXEL scheme; neutron data for unconstrained $\mathrm{COOH}$ groups are about 1.005-1.010 $\AA$;

3) setting $\mathrm{BCD}=114^{\circ}, \mathrm{BCF}=\mathrm{DCF}=123^{\circ}$ and $\mathrm{CDE}=110^{\circ}$.

A tentative .oih file ${ }^{2}$ is prepared using Retcif module on the reset.cif file (resetorig.oih), then the oih files are corrected inserting $\mathrm{O}$-atom and $\mathrm{H}$-atom generation lines with the specified bond distances, bond angles and torsion angles.

The corrected structures are: AMBNZA, BENZAC01, BIDLOP, DUTREQ, IKAYEZ, JOZZIH, TEPHTH12, TEPHTH13, WEBNAU, ZZZMUY01, COVJIG01 and ZZZMVI. 
Table S1. Labels in the NOCOR database.

ABEKUN02, ABELEY, ABELIC, ABORAM, ABUHIO, ABULEQ, ACEQUW, ACERAD, ACESEI, ACESIL, ACETAF, ACETEJ, ACNTCB, ACNTCQ, ACOGII, ACOMUC, ACONIR, ACOYOG01, ACOYUM, ADECIW, ADETOT, ADULEQ03, AFIGED, AFIYOF, AHASUX, AHATEI, AHATIM, AJAGIB, AJAGOH, AJAHEY, AJAHIC, AJAHOI, AJAHUO, AJAKAX, AJAKEB, AJAKOL, AJAKUR, AJALAY, AJIWIA, AKENEK, AKOTOJ, ALLCAM, AMBACO07, AMBSAM10, AMILEN, AMILUD01, AMIMAK, AMIMEO, ANOLIZ, ANOLOF, ANOLUL, ANOMAS, ANOTAZ, ANTCYB14, ANTPIC, ANTPML01, ANUNED, APANBZ, APEGEI, ASAKIO, ASAKOU, ASAVUL, ASAXOH, ASAXUN01, ASAYEY, ASIJER, ASIZEI, ASKNBZ, AWIHOE, AWUDEB, AWUQUF, AWUVEU, AXUFIJ, AXUFUV, AXUGOQ, AYOROW, AYOWER, AYUMEN, BACWUX10, BADCOY10, BALNAD10, BAPMAH, BARBAM06, BARBUR06, BARIMZ10, BARMIM, BARMPN, BATDUX, BAVVEZ, BAYVUS, BAZDAH, BECNUSO2, BEFGIC, BEJGUT, BEMLOV, BEPPAN, BEPYOK, BERMOA, BERNAN, BERNER, BERPET, BERQAQ, BERQEU, BERQIY, BEYVAD, BICVUE01, BIFYOE, BIHZAU, BILXUP, BIMSUM, BIQNEW, BIRDIP, BITROL, BIVSIJ02, BIWVUY, BIXFOD01, BIZYOA, BODSAO, BOGGUZ10, BOMBOW, BOMBUC, BOMGUH, BOQHOE, BOQNIG, BOQNUS, BORNUS, BORPAA, BORPII, BOWKAA, BPPFBP, BSXDMF, BUDWAY10, BUDWEC, BUHSIG, BUNRAD01, BUYSET10, BZPCBQ, BZPPAM01, BZQTCQ10, CACGUK, CAFWAH, CAHLON, CAHXQX, CAKYOE, CAKZEV, CANANP, CANLUY, CAPKUB, CAPRES, CAPTOC01, CARTEW, CATGIP, CATGOV, CAVYED, CAVYIH, CAXNOE, CBZTNB, CDSCDS, CECPEF, CEDSEL, CEGGUS, CEHCAV, CEHGEB, CEJTAM, CEKBUP, CEKFAB, CEKKOU01, CEKYUM, CERXIH, CERZEF, CEVPOK13, CEWYIM20, CEWYOT, CHRFAN, CHRTCQ01, CIDDAV, CIFWUJ, CIKCEF, CIKRAP, CILLAM, CILLOA, CILLUG, CILQAR, CIRMUN, CIRNAU, CIRNEYO1, CIRNIC, CIRWEG, CIRXAD, CIRXIL, CISCOW, CITSAZ10, CIWDAO, CIYDAR, CIYDEV, CIYDIZ, CLAHMB02, COFBUU10, COFCEF10, COGJOZ, COGKOA, COGREV, COHFEM, COHWIF01, COLHAN, CONZEK01, CORPIJ, COXLEG, COZXIZ, COZXOF, COZYIA01, COZYOG, COZZAT, COZZEX, COZZIB, COZZOH, CUFFAK, CUKVEK, CUMRAE, CUVDON, CUVDUT, CUWZAW, CUZDAC, DALPOX, DALPUD, DALQAK, DAQYOK, DAQYUQ, DAQZEB, DAQZIF01, DATFIP, DATQEV, DATQUL, DAVPAS, DAVPEW, DAYZOT, DBTTNB, DEHSIS10, DELGEG, DEVFOZ, DHBPHT10, DICCUP, DIFDOM, DIFNUC, DIJVOH, DIJVUN, DILTUP, DILVAX, DILVEB, DIMSUP, DINRUP01, DINSOK, DIPHUH, DITHUK, DITJAS, DITJEW, DIVVAF, DKPSAL10, DMAFBZ01, DMPTCN, DNPCPH, DOCWUN, DOKGUG01, DOKHAN, DOLCAJ, DOMHAQ, DOMHEU, DOPNAZ, DOPNED, DOQWUD, DOWQIQ, DTHOLC, DUCROJ, DUDCOU, DUHSEF, DURYUK, DURZAR01, DUTSOB, DUVHOR10, DUXZAX, DUZPEU, EADBAR10, EADPBA, EBIBEW, EBILOR, EBILUX, EBIMIM, EBONUG, ECELON01, ECUTUR, ECUVIH, ECUVON, EDAPOQ, EDAWAH, EDCDMP10, EDECOF, EDELUV, EDOROE, EFAPUY, EFAWUF, EFOZAB04, EFUCEP, EGENIP, EGIQAO, ELEGUY01, ENAGEG, ENAGIK, ENAHAD, ENAHIL, ENICIO, ENICOU01, EPUPUB01, EQISIH, EQISON, ERAVAU, ESATOH, ESATUN, ETABAC, EVANAR, EVETAB, EWAMUL, EWANAS, EWANEW, EWAPAU, EWAPIC, EWAQID, EWAQOJ, EWAQUP, EWARAW, EWEVOS, EWEVUY, EWOZIZ, EWUYOL, EXAMUM, EXANAT, EXANEX, EXANIB, EXAPEZ, EXAPID, EXEYIQ, EXEYOW, EXEYUC, EXEZEN, EXEZIR, EXEZOX, EXEZUD, EXIBAP, EXIBET, EXIBIX, EXIBOD, EXIBUJ, EXICAQ, EXICEU, EXICIY, EXUQUJ, EYIXUF, EYOLOU, EYULUG, EZEDAP, EZEGIA, EZIDUN, EZIFAV, EZIFID, EZOPEP, FABWOV, FADCES, FADGEY, FADGIC, FADGOI, FADGUO, FADHAV, FADHEZ, FADHID, FADHUP, FAFSEL, FAFSIO, FAFTAH, FAFTEL, FAGDOI, FAHLEF01, FAKMOV, FALSIW, FARMAM, FARQAR, FASJUG, FAVFIT, FEHREQ, FEJDUT, FENPOD, FENPUJ, FEPKES, FEPKIW, FEQXEF, FEQXIJ01, FERYAD, FERYEH, FERYIL, FERYOR, FESRAW, FEVZUD, FEZPAB, FICYIB, FICZIC, FIDCIG, FIHYEA, FILHIR, FILHOX, FINTAZO3, FINTIH, FITHIZ, FITJAT, FITJEX, FITLID, FITMEA, FITNIF, FIVNIJ, FIZFUR, FLSCAC, FOACAM, FOJGOC, FOJGUI, FOJSAY, FOKHUI, FOMQUU, FOMWIN, FORBAQ01, FOTYET, FOVPIQ, FOWSAM, FOXNOW, FOZHAD, FUDTUT, FUHGUL, FUHPAA, FURAOX, FUREHX, FUXYEC, GABZEP, GABZUF, GACZUF, GADGUN, GADQAD, GADQEH, GADQIL, GAFTUE, GAHFAX, GAHFIF, GALBIF, GALPIT, GALRIU01, GANXUP, GANYAW, GANYEA, GAPWID, GAUTAM15, GAVHER, GAVHUH01, GAWJEU, GAWLOG, GAWSAY, GAXRUU, GAXSAB, GAZWUB, GEBREK, GEFHEF, GEHROB, GEHTUJ, GEJLEN, GEKXEZ, GENLIV, GENLOB, GENLUH, GEPQUP, GEQRIF, GEQROL, GEQXIL, GERWEF10, GERYOR, GERZUA, GESBAJ, GESBEN, GESBIR, GESCAK, GESCUE, GESSAY, GETYIO, GEXMED, GEXMIH, GEYFAR, GEYSAE, GIDLUB, GIFMUD, GIGCEE, GIGWOI, GIJBEG, GIKKAM, GIMZUY, GINKUK, GIPQAX, GIPQEB01, GIQRON, GIRDAN, GIRDER, GIRDIV, GIRPON, GIVZUG, GIYHUS, GLUCUR20, GODNAO, GODQIZ, GODTUO, GOGQID, GOGYUX, GOHYUZ, GOJZUC, GOKBAL, GOKCEQ, GOLVIM, GOLVOS, GOLVUY, GOLWAF, GOMDET, GONBAM, GOPKUR, GOPLAY, GOTTEO, GUCQUQ, GUCREB, GUDREC, GUFDOB, GUHTUZ, GUKWOZ, GUQHUW, GURNEN, GURNIR, GURNUD, GUTNOY, GUTRUI, HADKUTO1, HAFVEQ, HAGYEU, HAJQOZ, HAJQUF, HALHOT, HARJOB, HAVHIV, HAYYOW, HEBFAX, HEBFEB, HEBFOL, HEDRIT, HEDROZ, HEZSIO, HEZSOU, HEZTAH, HEZTEL, HIBFED, HIBFIH, HIBFON, HIBGII, HIBGOP, HICTOD, HIDQOC, HIDRET, HIDRIX, HIDWAT, HIGPUJ, HIHPEU, HIJBOR, HILJAP, HIMGAL, HIMLUL, HINQUR, HIPMIC, HISKOL, HIVHIE, HIYCAT, HMTFCQ, HMTOFAO1, HOCCEJ, HODMLN, HOFJOC, HOGFEQ, HOGFOA, HOGFUG, HOGGAN, HOGGER, HOGGUH, HOJKEY, HOJLIC, HOLJAU03, HOMXOW, HONFIB, HONSOT, HONTAG, HONTEK, HONVAI, HOPJOM, HOPKIH, HOQGIF, HOQGOL, HOQGUR, HORVOA, HORVUG, HOVLEJ, HOWLAH, HOZBOO, HUFZUE, HUHQUW, HUHRAD, HUMYOE, HURYIC, HUSTOE, HUSTUK, HUSVIA, HUZCUA, HUZSEA, IACNCA, IBAPUW, IBEWAO, IBUJAQ, ICUMAW, ICYTINO1, IDAPUZ, IDAQAG, IDENEM, IDILAI, IDUBUF, IFACUO, IFIMAL, IFISOH, IFORAY, IFUQAC, IJETOG02, IJEZUT, IJOYAI, IKANAK, INACOQ, IPEVUV, IPEWEG, IPEWIK, IPUPIT, IQACIN, IQIHEW01, IQUNUE, IREKAR, IRUFIL, IRUGEI, IRUGIM, ISIJEA, ISIJIE, ITIXUE, IVOBOK, IWERAE, IWEREI, IXOVUN, IXOXEZ, IZIHEE, JAKPIV, JATMEW, JAWVAF, JAWVEJ, JAWVIN, JAWVOT, JAWWAG, JAXSOR, JAZBAO, JAZBES, JECZAS, JEDLAG01, JEGBON, JEJPIY, JELSEY, JESMAW, JESMEA, JESMOK, JESVIN, JICTIY, JILZOU01, JIMDUE, JIQCAN, JIQCIV, JOCMOD, JOCRIC, JOCVEC01, JOFJUK, JOKJEA, JOLCIY, JOLCOE, JOLSAE, JOZZED, JUBFER, JUDNAX, JUDWOU, JUFDUJ, JUFFAR, JUKJII, JUKJOO, KABLEG, KARCIQ01, KARHAM, KARHOA, KASYEJ, KATBAJ02, KATLAT01, KAXRAE, KAXRIM, KAXROS, KAXRUY, KAXSEJ, KAXSIN, KAXSOT, KAYFOH, KAYFUN, KAYGIC, KAYHAV, KAYHID, KAYHOJ, KEBJIM, KEFBAZ, KEFBED, KEFCAA, KEFCEE, KEGXOJ, KEKGAJ, KEKGUD, KEKHIS, KEMCEL, KEWNOQ, KEWZUI, KEXZIX, KEXZOD, KIBQOC, KIDXECO2, KIGKER, KIGKOB, KIGLAO, KIGLES, KIGLOC, KIGLUI, KIGNEV, KIGNIZ, KIHXAB, KIHXEF, KIHXOP, KIHXUV, KIHYAC, KIHYEG, KIHYIK, KIHYUW, KIHZAD, KIJLOG, KIJSED, KIJTEE, KINVAG, KIXVES, KIZVAQ, KIZYAV, KIZYEZ01, KIZYID, KIZZAW, KOBFIQ, KODXUY, KOHYAJ, KOLSEK, KONZEU, KOPJUW, KOPKEH, KOYNER, KOYPIX, KPIPFA10, KULMUA, KURGOU, KUZZUB, LABJIH, LABZUJ, LACDIC, LAGNAI, LAKXAW, LANYIK, LAPSAX, LAPSEB, 
LAPTUS, LAPVAA, LASGOD, LASGUJ, LATGEU, LATGIY, LATGUK, LATHEV, LATHIZ, LATJAT, LATJEX, LATKEY, LATKOI, LATKUN, LATKUO, LATLAU, LATLAV, LATLEY, LATLIC, LATQII, LATRAB, LATREF, LATRIJ, LATRUV, LATSEG, LATTAD, LATTIL, LATTORO1, LAWKIE, LEDJIO, LEDYUQ, LEFKAK, LEFMEP, LEHMIV, LEJROH, LENGOA, LEQXAG, LERXUD, LETBIX, LETSAG, LEWPUY, LEWREK, LEWRIO, LEWTAK, LEXQEM, LEXQIQ, LEZQUE, LICJUD, LIDVAW, LIGYOR, LIKSAZ, LINFIX, LIPWOW, LIPWUC, LIPXUD, LIQFAU, LIYSIV, LIZVUN, LIZWIC, LIZWUO, LOCHOB, LOCVOO01, LOFKIB, LOFLAV, LOFLID, LOGZIR, LOLSUA, LOMHUR, LOMTAI, LOMTEM, LOWJUC, LUDFUL, LUDYOZ, LUJSIT, LUKXUL, LUNMEM, LUNMIQ, LUNMOW, LUNNAJ, LUNNEN, LUNNOX, LUNNUD01, LUNPAL, LUNPEP, LUNRER, LUNYAU, LUNYEY, LUNYIC, LUNYOI, LUNYUO, LUNZAV, LUNZEZ, LUNZOJ, LUNZUP, LUTDUR01, MACLIM, MAGZIE, MAHKAJ01, MAHWUO, MAMPUM02, MAWPUW, MEBRAN, MEBRER, MEBRIV, MEBRUH, MECHAF, MECTUL, MECVEX, MECVIB, MECWOI, MECXID, MEFMAM, MEHFOV, MEWTAL, MIJTEG, MIPWEN, MIRQEJ, MIZMIT, MOCCEM, MODDIU, MODPAX, MOFROO, MOGVOV, MOGVUB, MOGWAI, MOGWEM, MOJCOF, MOKQUZ, MOPYAR, MOQGIJ, MORWAS, MOTRES, MOXVAX, MOXVIF, MOXVOL, MOXWAY, MOZCOU, MTHMAD13, MTZICA, MUDSAF, MUDSEJ, MUDSIN, MUFNIK01, MULYOI, MUQKOZ, MUROXA, MUSPAS, MUYTAC, MUYTEG, MXTTCQ01, MYFLTC, NABCAV, NABCEZ, NANFIR, NANPUO, NANQAV, NANQEZ, NANQOJ, NAPTIJ, NAPTOP, NAPYMA01, NARWAG, NAXSOW, NAXYOC, NAYMOP, NEBVOH, NEBWUO, NEBXAV, NEBXEZ, NEBXID, NEBXUP, NEHJER, NEHPIZ01, NELTIH, NEMHIW, NEMSUT, NEMTAA, NEMTEE, NEMTOO, NENDAM, NEPXEN, NEPXIR, NERNUT, NEWRUD, NEWXAO, NEXWUJ, NEXXIX, NEZXAT, NHAZMSO2, NIBJUF, NIFCAH, NIGFIS, NIGNIB, NIGNOH, NIJGIY, NIJGOE, NIJHAR, NIJHEV, NILZOX, NILZUD, NIMBAM, NIMGARO1, NINXAL, NINXOZ, NIPTUC, NIQNIL, NISLOQ01, NISMAD, NISTAK, NITGUS, NITRIR01, NIVYOG, NIWCIF, NOBYAE, NOKDAS, NOKWUF, NOLUU, NONZOF, NONZUL, NOPDAY, NOQFUV, NOVSIA01, NOVSOG, NOVSUM, NOVTEX, NPOAPL, NPOFNP, NPQHRQ, NUBHOH, NUCQEH, NUCQIL, NUDLON, NUFSIQ, NUGQOW, NUGZEV, NUGZOF, NUHXUJ, NUHYEU, NUHYIY, NUHYOE, NUHYUK, NUJDOL, NUJXEW, NUKWEW01, NUKWOG, NUKXAT, NUKXEX, NUKXUN, NUKYAU01, NUKYEY, NUKYOI, NUKYUO, NUKZAV, NUKZEZ01, NUKZID, NUKZOJ, NUNMOY, NURHIR, NUSVAZ, NUWGUH, NUYBUF, OBUBOE, OBULUU, OCAMUC, OCATUH, OCAYIA01, OCAYUM, OCAZAT, OCEBUV, OCEGEK, OCIPOG, OCIPUM, ODOBIT01, ODOHEV, ODOHIZ, OFIYOT, OFIYUZ, OFIZAG, OFOKOK, OFUGUR, OFUHIG, OFUHOM, OFUYIZ, OGAFOS, OGAFUY, OGAGUY, OGAHAF, OGAHEJ, OGAREV, OGENAQ, OGEPIA, OGEPOG, OGEZIJ, OGEZOP, OHIQUS, OJENIA, OKIRAB, OMESOP, ONAPUP, ONAQAW, ONAQIE, OQOMOX, ORASEG, ORAWIO, ORUXOP, ORUXUV01, OTAZUF, OTESAI, OTESEM, OTIYUM, OTIZAT, OTIZEX, OTIZIB, OVEZUL, OVIBAX, OVIBEB, OVIJIN, OVIVAR, OVUFUH, OYEREQ01, PABBAV, PABNIR, PAHHOW, PAHHUC, PAHYUT, PAJBIM, PAKNUM, PAMWUX, PANCYQ, PANKAR, PANYIM, PAPFOB, PAQNOM, PAVGOK, PAVTAI, PAVXAN, PAVYIW, PDTCNB, PECQOF, PECREW, PEFGEO, PEFGIS, PEHFUF, PEKREE, PEKRUU, PEKTAC, PEKWUZ, PEKXAG, PEMBAL01, PEMBEE, PENPYM, PEQPAD, PEQQIM, PESPEJ, PESROV, PEZPUF, PEZQAN, PHNSNB12, PIBDAF, PICDUA, PICFAI, PICFUM, PIDKAQ, PIDKEU, PIFHAO, PIKMIF, PILKEC, PILKOM, PILKUS, PILLAZ, PIRNOV01, PIRPEN, PIRPIR, PIRQEO, PIRQIS, PIRREP, PIRXOF, PIRXUL, PIRYAS, PITYAS, PITYEW, PITYIA, PITYOG, PITZEX, PIWKEL, PIWXOI, PIWXUO, PMEANT, PMPZOL04, POBDAL, POCPED, PODLAV, POFVOV, POFWUC, POHDAR, POKBOI, POSREV, POVYEF, POVYIJ, POVYOP, POVYUV, POVZAC, POXGAL, PTZPMA, PTZTCQ01, PUFFUS, PUHFAA, PUHROZ13, PUJBOM, PUJHUY, PUJNIS, PUKPER, PUMQET, PUMVOI, PUMVUO, PUMWEZ, PUMWID, PUNYUS, PUPGUD, PUQGEO, PUVMOI, PUWFET, PUWFIX, PUYTAE, PVVBFD01, PYOCHP, PYRBZQ01, PYRCBZ02, PYRFLR01, PYRPMA02, PYRTCQ02, QACVAT, QAFFIM, QAHSIB, QAKRIF, QAKSUS, QAMQUR, QAMRAY, QAMROM, QAMRUS, QAMSAZ, QECYII, QEGKAO, QELYAI, QERCAS, QETMOS, QETMUY, QETNAF, QEVMEJ, QEWHEH, QEWHIL, QIGVEH, QIGVIL, QIJCUH, QILZOA, QIMHOJO1, QIMHUP, QIRKUZ, QIRLAG, QIRLEK, QIRNUC, QOBCOB, QOFXUE, QOGMII, QOLMAH, QOLMEL, QOLMIP, QOLMUB, QOLNAI, QOLNEM, QOLNOW, QOLSAM, QONPUE, QONYUP, QOQJOX, QOQKEO, QOQKOY, QOQPOD, QOTCEH, QOVHOZ, QOVHUF, QQQAMSO2, QUBQAF, QUBQEJ, QUBQOT, QUETHU, QUINAC, QULLUF02, QUNQUM, QUOLUR, QUTFAN, QUWZEO, QUWZIS, QUWZOY, QUYJEA, RADDOQ, RADFEI, RADGAF, RAHHEN, RAWCOH, RAWCUN, RAYLOS, RAZPUE, REBXON, REBXUT, REDCIM, REDCUY, REDDAF, REDDEJ, REDFAH, REFCEK, REGKIX, REGKUK, REGWIJ, REHMUM, REHNAT, REHSAA, REHSEE, REHSII, REHSUU, RENPUV, REQWAM, RESFOL, RESGAY, RESGIG, RESGOM, RESHAZ, RESHED, RESHIH, RETZEW, REXNAL, REYPIU, RIDFAN, RIDJOD, RIDKUK, RIDLEV, RIDPAV, RIDPEZ, RIFQAY03, RIGDES, RIHBUG, RIJNEF, RILJEB, RILOL, RINHUT, RIRPOX, RIWGAH, RIWJIQ, RIYXUT, RODSEK, ROGKOO, ROJCEA, ROKNAH, ROKQAJ, ROKQEN, ROLFUU, RUCDUP, RUCFAX, RUJCII, RURROM, RUVVAF, RUWDUJ, RUWFAR, RUWFEV, RUWFOF, RUWGEW, RUWGIA, RUWHIB, RUWMIF, RUXDAP, RUXLUS, RUXMAZ, RUXMED, RUXMIH, RUXMON, RUXMUT, RUXNAA, RUXQEG, RUXQUW, RUXRAD, RUYGIC, RUYHEZO1, RUYKUR, RUYLAY, RUZMOO, SABCEF, SABDOQ, SACCAF01, SACSOE, SADTIC, SADTOI, SAFNOE, SAGQEW, SAHHEQ, SAKLAR, SALCYS, SAMTEF, SANAPY, SARDAQ, SARDIY, SARDOE, SARJUQ, SARLEC, SAXHIK, SAXPAK, SAYNEM, SAYNIQ, SAYNOW, SAYPAL, SAZKEK, SEDJUI, SEDMEV, SEDNIY, SEGFEP, SEGFUG, SEGHUI, SENJOK, SENYIV, SEQVIV, SEQZUK, SERASC10, SERMOR10, SESLIM, SETRIU, SETROA, SETSAN02, SIBGUF, SIBHEQ, SIDHUK, SIDJEW, SIDJIA, SIDSEF, SIHRAE, SIJZOC, SIJZUI, SILKIH, SINTAK, SIQLOU, SIRBUR, SITCUU, SITDIJ, SIVBAA, SIXFAH, SIXYAZ, SIYCIN, SLCADC10, SOBNEE, SOCMEE, SOCMII, SODDOF01, SOGXUH, SOHXAQ, SOJPEM, SOLBECO1, SOLFAC, SOLFEG, SOLFIK, SOLFUW, SOLGAD, SOLHUY, SOMHEI, SOMKEL, SONCED, SOQPAQ, SORBPY20, SORWEB, SORWIF, SOVBOU, SOVFOY, SOVHEQ, SOWLOF, STHSAM01, SUGZUQ, SUHBED, SULFZC, SULTHE01, SUTVAF, SUVJEY, SUVZEO, SUWGOG, SUXVOW, SUYWEP, SUZQIO, SUZQOU, SUZZOC, TACCAD, TACCIL, TACCUX, TACRUL, TACSAS, TACSEW, TAKLEX, TAMZAK, TARLAA, TASLAA, TASLEE, TAWDOL, TAWNEL, TAWSOB, TAZWOH, TCQBDX, TCQMHP, TCQNAP01, TCQPDA, TCQPEN10, TECCAF02, TECSEZ, TEHNAW, TEJGUK01, TEKKID, TELVIP, TENNOR, TENSIQ, TENSOW, TENYAO, TENYES, TERSAK, TETXUL, TEWKOV, TEXPOB10, TEZNET, TEZNIX, TEZNOD, THLCTC, TIBZIO, TICFOA, TICREE, TICROO, TICYIP03, TIDQAZ, TIGNEE, TIJKIG, TIJMUU, TIJTUB01, TIJVAJ, TIPFII, TIPWIY01, TIVCOS, TIVNUI, TIWZAA, TMPASD, TNIZMS, TOBSOU, TOKJUY, TOMWOJ, TOMXEA, TONGOS, TPDTCB01, TTFCAN14, TTFFAN, TUHJAH, TUPRBN01, TUQBIR, TUWWEO, UCOTIQ, UDEKEV, UDEKIZ, UDEQUP01, UDUZEY, UDUZIC, UDUZOI01, UFETAA, UHACEM, UHACIQ, UHACUC, UHADOX, UHADUD, UHAFAL, UHAFEP, UHAPUO, UHAQAV, UHAQEZ, UHAQID, UHAQOJ, UHAQUP, UHEMEZ, UHEVIN, UHORUF, UHOSAM, UHOSEQ, UHOSOA, UHUNAM, UJORAM, UJOREQ, UKOKUA, UKOSAPO1, UKOSET, UKOSIX, ULAWAF, ULAWUZ, ULAXAG, ULAXEK, ULAZEM, ULAZIQ, ULECEU, ULEKIG, ULEWIS, ULOCII, ULUTEA, UMINAF, UMINOT, UMIQIR, UMUZAE, UNEBOE, UNEFUO, UNEGAV, UNEGEZ, UNEZAO, UNEZES, UNIRIT, UNISUG, UNUQOK, UPEPUB, UPOQEW, 
UREXPO11, URIJEL, URIKAI, URIKEM, URIKUC, URILAJ, URILEN, URILUD, URISAQ, URMALN, URNFRO, URODEL, URODIP, UROXAL01, UROXAM, URPRBN01, USOBOU, UVIMES, UXOFOD, UYOTIM, UYOTUY, VABVOL, VAFWOP, VAKTOR, VAKTOS, VAKTUX, VAKVIN, VATCOI, VAWKIO, VAWLAG, VAWLEK, VAXQUI, VAXRAP, VAXVOH, VAXWAU, VAZBIJ, VEDFAM, VEHDIW, VEHNIF, VEJXAJ, VEMKON, VEMZIX, VENLUV, VENZOD, VEQKIL, VERJUY01, VESCUS, VEVGAG, VEVGEK, VEVGIO, VEVGOU, VEVGUA, VEVYOM, VEXQOE, VEXQUK, VEXWUQ, VIDHOG, VIFFEV, VIFKUR, VIGFAT, VIGFEX, VIGFIB, VIGFOH, VIGFUN, VIGGEY, VIGGOI, VIGKIF, VIHGOI, VIJTIR, VIKLOR, VIKLUX, VILSEP, VIPVIB, VIPXUP, VITKEP, VOCJAY, VOCTEO, VOCZET, VOHKUA, VOJGIK, VORCOV, VORLOE, VUHFIO, VUHKEO, VUPQUT, VUPRAA, VUPREE, VUPRII, VUPROO, VUQVUZ, VUXRAI, VUYVIV, VUZMEJ, VUZNIO, VUZZEW, VUZZIZ, VUZZOF, WABGEL, WABWEB, WADTOM, WAFFUG, WAFNIB, WAFNOH, WAFNUN, WAGMOH02, WAJVOU, WAJWEL, WAJXAI, WANCOE, WANDEV, WANJOM, WATREPO4, WAWDEE, WAWPAM, WEBQAX, WEDKUN, WEDLAU, WEFKOJ, WEMHEB, WEPDIF, WEXQIA, WEYFEN, WEYROH, WEZXUW, WICZUF, WIFZOC, WIGCEW, WIGNIL, WIKTEP, WINMIQ, WINTIW, WIQNIV, WIRWID, WOBJOM, WOBQEK, WOBQIO, WOCFAX, WOCHIH, WOCHON01, WOCVIT, WOJGUX, WOJHAE, WOJHEI, WOJHEJ, WOLXEA, WOPCOV, WOPDEM, WOPPOH, WOPZUW, WOQBAF02, WOQBEJ, WOQBIN, WOQBOT, WOQBUZ, WOWFET, WUBYUN, WUDQAN, WUKREZ, WURNON, WURNUT, WUVKEE, WUYROX, WUZHOP, XADVED, XAGFER, XAHZAH, XAMTOV, XAMWEO, XAPMAC, XAPMIK, XAPWOA, XAPWUG, XAQQEM, XAQQIQ, XAQQOW, XAQRIR, XAQVES, XAQVIW, XASCUP, XASDEA, XASDIE, XAXPIV02, XEBFEQ, XEBFOA, XEGHEW, XEJWUF, XEJXAM, XEJXEQ, XEMBOI, XEMBUO, XEMCAV, XEMCID, XEPBEB, XEPBIF, XEPBOL, XEPCAY, XEQCUT, XEQDAA, XESXOJ, XETLIS, XEWTUO, XEWVAW, XEXCEI, XIFQEJ, XIHNOS, XIPTOG, XISCUZ, XISDAG, XISDEK, XIWLEU, XIYWIM, XOBCAT, XOBCEX, XOBCIB, XOBCUN, XOBHIF, XODPIR, XODPOX, XOGFOQ, XOGMIR, XOGMUD, XOGNEO, XOGPAM, XOHXAU, XOJMUF, XOJNAM, XOJNIU, XOJNOA, XOKBOO, XOKSUN, XOKTIC, XOLHOW, XOLHUCO2, XOMZEG, XONBAF, XONBEJ, XONBIN, XONBOT, XONBUZ, XONCIO, XONPUN, XONQIC, XOXHEY, XOZJAY, XUDCII, XUGPEU, XUNGIW, XUNGUI, XUNHIX, XUNHOD, XUNHUJ, XUNJAR, XUVPIO, XUVPUA, XUVQIP, XUVQOV, XUVQUB, XUVSA, XUVTEO, XUVVAM, YAFZAGO1, YAGFAP, YAHFOC, YAHQEF, YASGAC, YASGEG, YASGIK, YASGOQ04, YATBOM, YAWQOD, YAXWOL, YECHAQ, YEJYOB, YEPCOM, YIZXEL, YIZXIP, YOCZIB, YOCZUL, YODYAR, YOLIS, YOLQOF, YORJOF, YORREC, YOSMOI, YUCPIW, YUGLUI, YUPWOW, YUQHEY, YURTEL, YUWNEJ, ZABRAV, ZAGKOH, ZAGKUN, ZAJHOH, ZAJJAV, ZAJJEZ, ZAQSOB, ZAQTUG, ZAVXAV, ZAYQEV, ZEBXOV, ZEBXUB, ZEFFEX09, ZEFVAJ, ZEVSIC, ZEVWEE, ZEXTIF, ZEXVUV, ZEXWEG, ZEXWIK, ZEYNOI, ZEZHAP, ZEZHET, ZEZHIV, ZIBCEU, ZIJQOY, ZIKNOY01, ZIKPUG01, ZILLUB, ZILMAI, ZIMHIO01, ZINZUR, ZIPBAB, ZODWIY, ZOGCOP, ZOHTEX, ZOHTIB, ZOHVUP, ZOHWAW, ZOHWEA, ZOHWIE, ZOHWOK, ZOHWUQ, ZOHXAX, ZOKYON, ZOKYUV, ZUPKUQ, ZUPLAX, ZUPLEB, ZUTLUV, ZUWKUX, ZZZAGV01, ZZZAKD10, ZZZGKE01, ZZZGMW02. 
Table S2. Labels in the DIM database.

ABEKUN02, ABELEY, ABELIC, ABORAM, ABULEQ, ACEQUW, ACERAD, ACESEI, ACETAF, ACETEJ, ACNTCQ, ACOGII, ACOMUC, ACONIR, ACOYOG01, ACOYUM, ADETOT, ADULEQ03, AFIGED, AFIYOF, AHASUX, AHATEI, AHATIM, AJAGOH, AJAHEY, AJAHIC, AJAHOI, AJAHUO, AJAKAX, AJAKEB, AJAKOL, AJAKUR, AJALAY, AJIWIA, AKENEK, AKOTOJ, AMBACO01, AMILEN, AMILUD01, AMIMAK, AMIMEO, ANOLIZ, ANOLOF, ANOLUL, ANOMAS, ANTCYB14, ANTPIC, ANTPML01, ANUNED, APANBZ, APEGEI, ASAKIO, ASAKOU, ASAVUL, ASAXOH, ASAXUN01, ASAYEY, ASIJER, ASIZEI, ASKNBZ, AWIHOE, AWUQUF, AWUVEU, AXUFIJ, AXUFUV, AXUGOQ, AYOROW, AYOWER, AYUMEN, BADCOY10, BALNAD10, BAPMAH, BARBAM06, BARBUR06, BARIMZ10, BARMIM, BARMPN, BAZDAH, BECNUSO2, BEJGUT, BEMLOV, BEPPAN, BERMOA, BERNAN, BERNER, BERPET, BERQAQ, BERQEU, BERQIY, BEYVAD, BICVUE01, BIFYOE, BIHZAU, BIMSUM, BIQNEW, BIRDIP, BITROL, BIVSIJ02, BIWVUY, BIZYOA, BODSAO, BOMBOW, BOMBUC, BOQHOE, BOQNUS, BORNUS, BORPAA, BORPII, BOWKAA, BPPFBP, BSXDMF, BUDWAY10, BUDWEC, BUHSIG, BUNRAD01, BZPCBQ, BZPPAM01, BZQTCQ10, CACGUK, CAFWAH, CAHLON, CAKYOE, CAKZEV, CAPTOC01, CARTEW, CATGIP, CATGOV, CAXNOE, CBZTNB, CECPEF, CEDSEL, CEGGUS, CEHCAV, CEKBUP, CEKFAB, CEKKOU01, CERXIH, CERZEF, CEVPOK13, CEWYIM20, CEWYOT, CHRFAN, CHRTCQ01, CIFWUJ, CIKCEF, CIKRAP, CILLAM, CILLOA, CILLUG, CIRMUN, CIRNAU, CIRNEY01, CIRNIC, CIRWEG, CIRXAD, CIRXIL, CISCOW, CIYDAR, CIYDEV, CIYDIZ, CLAHMB02, COFBUU10, COFCEF10, COGJOZ, COGREV, COHFEM, COHWIF01, COLHAN, CORPIJ, COXLEG, COZXIZ, COZXOF, COZYIA01, COZYOG, COZZAT, COZZEX, COZZIB, COZZOH, CUKVEK, CUMRAE, CUZDAC, DALPOX, DALPUD, DALQAK, DAQYOK, DAQZEB, DAQZIF01, DATFIP, DATQEV, DATQUL, DAVPAS, DAVPEW, DAYZOT, DBTTNB, DEVFOZ, DICCUP, DIFDOM, DIFNUC, DIJVOH, DIJVUN, DILTUP, DILVAX, DINRUP01, DINSOK, DIPHUH, DITHUK, DITJAS, DITJEW, DIVVAF, DKPSAL10, DMAFBZ01, DNPCPH, DOKHAN, DOLCAJ, DOMHAQ, DOMHEU, DOPNAZ, DOPNED, DOWQIQ, DUCROJ, DURYUK, DURZARO1, DUTSOB, DUVHOR10, DUXZAX, DUZPEU, EADBAR10, EADPBA, EBILOR, EBILUX, EBIMIM, ECELON01, ECUTUR, ECUVIH, ECUVON, EDAPOQ, EDAWAH, EDCDMP10, EDECOF, EDELUV, EFAPUY, EFAWUF, EFOZAB04, EFUCEP, EGENIP, EGIQAO, ELEGUY01, ENAGEG, ENAGIK, ENAHAD, ENAHIL, EPUPUB01, EQISIH, EQISON, ERAVAU, ESATOH, ESATUN, EVANAR, EVETAB, EWANEW, EWAPAU, EWAPIC, EWAQID, EWAQOJ, EWAQUP, EWARAW, EWEVOS, EWEVUY, EWOZIZ, EWUYOL, EXAMUM, EXANAT, EXANEX, EXANIB, EXAPEZ, EXEYIQ, EXEYOW, EXEYUC, EXEZEN, EXEZIR, EXEZOX, EXEZUD, EXIBAP, EXIBET, EXIBIX, EXIBOD, EXIBUJ, EXICAQ, EXICEU, EXICIY, EXUQUJ, EYIXUF, EYOLOU, EZEDAP, EZEGIA, EZIDUN, EZIFAV, EZIFID, FADCES, FADGIC, FADGOI, FADGUO, FADHAV, FADHEZ, FADHID, FADHUP, FAFSEL, FAFTAH, FAGDOI, FAHLEF01, FARQAR, FASJUG, FAVFIT, FENPUJ, FEPKES, FEQXEF, FEQXIJ01, FERYAD, FERYEH, FERYIL, FERYOR, FEVZUD, FICYIB, FICZIC, FILHIR, FILHOX, FINTIH, FITHIZ, FITJAT, FITJEX, FITLID, FITMEA, FITNIF, FIVNIJ, FLSCAC, FOACAM, FOJGOC, FOJGUI, FOJSAY, FOMQUU, FOMWIN, FORBAQ01, FOTYET, FOVPIQ, FOWSAM, FOXNOW, FOZHAD, FUDTUT, FUHGUL, FUHPAA, FURAOX, GACZUF, GADGUN, GAFTUE, GAHFAX, GAHFIF, GALBIF, GALRIU01, GANYEA, GAPWID, GAUTAM15, GAVHER, GAVHUH01, GAWJEU, GAWLOG, GAXRUU, GAXSAB, GAZWUB, GEFHEF, GEHROB, GEHTUJ, GEJLEN, GENLIV, GENLOB, GENLUH, GEPQUP, GEQRIF, GEQROL, GEQXIL, GERYOR, GERZUA, GESBAJ, GESBEN, GESBIR, GESCAK, GESCUE, GEXMED, GEXMIH, GEYSAE, GIDLUB, GIFMUD, GIGCEE, GIGWOI, GIMZUY, GIPQEB01, GIRDAN, GIRDIV, GIRPON, GIVZUG, GLUCUR20, GODNAO, GODTUO, GOGQID, GOGYUX, GOHYUZ, GOKCEQ, GOLVIM, GOLVOS, GOLVUY, GOLWAF, GOMDET, GONBAM, GOPKUR, GOPLAY, GUCQUQ, GUCREB, GUFDOB, GUHTUZ, GUKWOZ, GUQHUW, GUTRUI, HADKUTO1, HAFVEQ, HAGYEU, HAJQOZ, HAJQUF, HALHOT, HARJOB, HAVHIV, HEBFEB, HEBFOL, HEDRIT, HEDROZ, HEZSIO, HEZSOU, HEZTAH, HEZTEL, HIBGII, HIBGOP, HICTOD, HIDWAT, HIGPUJ, HIHPEU, HIJBOR, HILJAP, HINQUR, HIVHIE, HIYCAT, HMTFCQ, HOFJOC, HOGFEQ, HOGFUG, HOGGAN, HOGGUH, HOJKEY, HOJLIC, HOLJAU03, HOMXOW, HONFIB, HONSOT, HONTAG, HONTEK, HONVAI, HOPJOM, HOQGIF, HOQGOL, HOQGUR, HORVOA, HORVUG, HOVLEJ, HOWLAH, HOZBOO, HUFZUE, HUHQUW, HUHRAD, HUMYOE, HURYIC, HUSTOE, HUSTUK, HUSVIA, HUZCUA, HUZSEA, IACNCA, IBUJAQ, ICYTIN01, IDAPUZ, IDAQAG, IDENEM, IDUBUF, IFACUO, IFIMAL, IFORAY, IFUQAC, IJEZUT, IJOYAI, INACOQ, IPEWEG, IPEWIK, IPUPIT, IQIHEW01, IREKAR, IRUFIL, IRUGEI, IRUGIM, ISIJEA, ISIJIE, ITIXUE, IVOBOK, IWERAE, IXOVUN, IXOXEZ, JAWVAF, JAWVEJ, JAWVIN, JAWVOT, JAWWAG, JAXSOR, JAZBAO, JAZBES, JECZAS, JEJPIY, JELSEY, JESMAW, JESMOK, JESVIN, JILZOU01, JIQCAN, JOCMOD, JOCRIC, JOCVEC01, JOFJUK, JOKJEA, JOLCIY, JOLCOE, JOLSAE, JUBFER, JUDNAX, JUDWOU, KABLEG, KARCIQ01, KARHAM, KARHOA, KATBAJ02, KAXRAE, KAXRIM, KAXROS, KAXRUY, KAXSEJ, KAXSIN, KAXSOT, KAYFOH, KAYFUN, KAYGIC, KAYHAV, KAYHID, KAYHOJ, KEBJIM, KEFBAZ, KEFBED, KEFCAA, KEFCEE, KEKGUD, KEKHIS, KEMCEL, KEWNOQ, KEWZUI, KEXZIX, KEXZOD, KIBQOC, KIDXEC02, KIGKER, KIGKOB, KIGLAO, KIGLES, KIGLOC, KIGLUI, KIGNIZ, KIHXAB, KIHXEF, KIHXOP, KIHXUV, KIHYAC, KIHYEG, KIHYIK, KIHYUW, KIHZAD, KIJLOG, KIJSED, KIJTEE, KINVAG, KIXVES, KIZVAQ, KIZYAV, KIZYEZO1, KIZYID, KIZZAW, KOBFIQ, KODXUY, KOHYAJ, KOLSEK, KONZEU, KOPKEH, KOYNER, KURGOU, KUZZUB, LABJIH, LABZUJ, LAGNAI, LANYIK, LAPSAX, LAPSEB, LAPTUS, LAPVAA, LASGOD, LASGUJ, LATGEU, LATGIY, LATGUK, LATHEV, LATHIZ, LATJAT, LATJEX, LATKEY, LATKOI, LATKUN, LATKUO, LATLAU, LATLAV, LATLEY, LATLIC, LATQII, LATRAB, LATREF, LATRIJ, LATRUV, LATSEG, LATTIL, LATTORO1, LAWKIE, LEDYUQ, LEHMIV, LENGOA, LEQXAG, LERXUD, LETBIX, LETSAG, LEWPUY, LEWREK, LEWTAK, LEXQEM, LEZQUE, LICJUD, LIGYOR, LINFIX, LIPWUC, LIPXUD, LIQFAU, LIYSIV, LIZVUN, LIZWIC, LIZWUO, LOCHOB, LOCVOO01, LOFKIB, LOFLID, LOGZIR, LOLSUA, LOMHUR, LOMTAI, LOMTEM, LUDFUL, LUJSIT, LUKXUL, LUNMEM, LUNMIQ, LUNMOW, LUNNAJ, LUNNEN, LUNNOX, LUNNUD01, LUNPAL, LUNPEP, LUNYAU, LUNYIC, LUNYOI, LUNYUO, LUNZEZ, LUNZOJ, LUNZUP, LUTDUR01, MAGZIE, MAHKAJ01, MAHWUO, MAWPUW, MEBRAN, MEBRER, MEBRIV, MEBRUH, MECHAF, MECTUL, MECVEX, MECVIB, MECWOI, MECXID, MEFMAM, MEHFOV, MEWTAL, MIJTEG, MIRQEJ, MIZMIT, MOCCEM, MODDIU, MODPAX, MOFROO, MOGVOV, MOGVUB, MOGWAI, MOGWEM, MOJCOF, MOKQUZ, MOPYAR, MOQGIJ, MOTRES, MOXVAX, MOXVIF, MOXVOL, MOXWAY, MOZCOU, MTHMAD13, MTZICA, MUDSEJ, MUDSIN, MUFNIKO1, MULYOI, MUQKOZ, MUROXA, MUSPAS, MUYTEG, MXTTCQ01, MYFLTC, NANPUO, NANQAV, NANQEZ, NANQOJ, NAPTIJ, NAPTOP, NAPYMA01, NARWAG, NAXSOW, NAYMOP, NEBVOH, NEBWUO, NEBXAV, NEBXEZ, NEBXID, NEBXUP, NEHJER, NEHPIZ01, NEMHIW, NEMSUT, NEMTAA, NEMTEE, NEMTOO, NENDAM, NEPXIR, NERNUT, NEWRUD, NEXWUJ, NEXXIX, NEZXAT, NHAZMSO2, NIBJUF, NIFCAH, NIJGIY, NIJHAR, NIJHEV, NILZOX, NILZUD, NIMBAM, NINXOZ, NIQNIL, NISLOQ01, NISMAD, NISTAK, NIVYOG, NIWCIF, NOKDAS, NOKWUF, NONZOF, NONZUL, NOPDAY, NOQFUV, NOVSIA01, NOVSOG, NOVTEX, NPOAPL, NPOFNP, NPQHRQ, NUCQEH, NUDLON, NUFSIQ, NUGQOW, NUGZEV, NUGZOF, NUHXUJ, NUHYIY, NUHYOE, NUKWOG, NUKXAT, NUKXEX, NUKXUN, NUKYAU01, NUKYEY, NUKYOI, NUKYUO, NUKZAV, NUKZEZO1, NUKZID, NUKZOJ, NURHIR, NUSVAZ, 
NUWGUH, NUYBUF, OBUBOE, OBULUU, OCAMUC, OCATUH, OCAYIA01, OCAYUM, OCAZAT, OCEBUV, OCEGEK, OCIPOG, ODOBITO1, ODOHEV, OFIYOT, OFOKOK, OFUGUR, OFUHIG, OFUHOM, OFUYIZ, OGAFOS, OGAFUY, OGAREV, OGENAQ, OGEPOG, OHIQUS, OKIRAB, ONAQAW, ONAQIE, OQOMOX, ORASEG, ORAWIO, ORUXOP, ORUXUV01, OTAZUF, OTESAI, OTESEM, OTIYUM, OTIZAT, OTIZEX, OTIZIB, OVEZUL, OVIBAX, OVIBEB, OVIJIN, OVUFUH, PABBAV, PABNIR, PAHHUC, PAHYUT, PAJBIM, PAMWUX, PANCYQ, PANKAR, PANYIM, PAPFOB, PAVGOK, PAVTAI, PAVXAN, PAVYIW, PDTCNB, PECREW, PEFGEO, PEFGIS, PEHFUF, PEKREE, PEKRUU, PEKTAC, PEKWUZ, PEKXAG, PEQQIM, PEZPUF, PEZQAN, PHNSNB12, PIBDAF, PIDKAQ, PIDKEU, PIFHAO, PIKMIF, PILKEC, PILKUS, PILLAZ, PIRNOV01, PIRPEN, PIRPIR, PIRQEO, PIRQIS, PIRREP, PIRXOF, PIRXUL, PITYAS, PITYEW, PITYIA, PITYOG, PITZEX, PIWKEL, PIWXOI, PIWXUO, PMPZOL04, POBDAL, POCPED, POFVOV, POSREV, POVYEF, POVYIJ, POVYOP, POVYUV, POVZAC, POXGAL, PTZPMA, PTZTCQ01, PUFFUS, PUHFAA, PUJBOM, PUJHUY, PUJNIS, PUKPER, PUMQET, PUMVOI, PUNYUS, PUPGUD, PUQGEO, PUVMOI, PUWFIX, PVVBFD01, PYRBZQ01, PYRCBZ02, PYRFLR01, PYRPMA02, PYRTCQ02, QACVAT, QAFFIM, QAKRIF, QAMQUR, QAMRAY, QAMROM, QAMRUS, QAMSAZ, QECYII, QEGKAO, QELYAI, QERCAS, QETMOS, QETMUY, QETNAF, QEVMEJ, QEWHEH, QEWHIL, QIGVEH, QIGVIL, QIJCUH, QILZOA, QIMHOJ01, QIMHUP, QIRNUC, QOBCOB, QOGMII, QOLMAH, QOLMEL, QOLMIP, QOLMUB, QOLNAI, QOLNEM, QOLNOW, QOLSAM, QOQJOX, QOQKEO, QOQKOY, QOQPOD, QOTCEH, QOVHOZ, QOVHUF, QQQAMS02, QUBQAF, QUBQEJ, QUBQOT, QULLUF02, QUWZEO, QUWZIS, QUWZOY, RADDOQ, RADGAF, RAHHEN, RAWCOH, RAWCUN, RAYLOS, RAZPUE, REBXON, REBXUT, REDCIM, REDDAF, REDDEJ, REDFAH, REGKUK, REGWIJ, REHMUM, REHNAT, REHSAA, REHSEE, REHSII, REHSUU, RENPUV, REQWAM, RESFOL, RESGAY, RESGIG, RESGOM, RESHAZ, RESHED, RESHIH, RETZEW, REYPIU, RIDFAN, RIDJOD, RIDKUK, RIDLEV, RIDPAV, RIDPEZ, RIFQAY03, RIGDES, RIJNEF, RILJEB, RILJOL, RINHUT, RIRPOX, RIWGAH, RIWJIQ, RIYXUT, RODSEK, ROGKOO, ROJCEA, ROKQAJ, ROKQEN, ROLFUU, RUCDUP, RUCFAX, RUJCII, RURROM, RUVVAF, RUWDUJ, RUWFAR, RUWFEV, RUWFOF, RUWGEW, RUWGIA, RUWHIB, RUWMIF, RUXDAP, RUXLUS, RUXMAZ, RUXMED, RUXMIH, RUXMON, RUXMUT, RUXNAA, RUXQEG, RUXQUW, RUYGIC, RUYHEZ01, RUYKUR, RUYLAY, RUZMOO, SABCEF, SABDOQ, SACCAF01, SACSOE, SADTIC, SAFNOE, SAGQEW, SAKLAR, SANAPY, SARDAQ, SARDIY, SARDOE, SARJUQ, SARLEC, SAXHIK, SAXPAK, SAYNEM, SAYNOW, SAYPAL, SAZKEK, SEDJUI, SEDMEV, SEGFUG, SEGHUI, SENYIV, SEQVIV, SERASC10, SERMOR10, SESLIM, SETRIU, SETROA, SETSAN02, SIBGUF, SIBHEQ, SIDIIA, SIDSEF, SIHRAE, SIJZOC, SILKIH, SIRBUR, SITCUU, SITDIJ, SIVBAA, SIXFAH, SIXYAZ, SIYCIN, SLCADC10, SOBNEE, SOCMEE, SOCMII, SODDOF01, SOGXUH, SOHXAQ, SOJPEM, SOLBEC01, SOLFAC, SOLFEG, SOLFIK, SOLFUW, SOLGAD, SOLHUY, SOMHEI, SOMKEL, SONCED, SOQPAQ, SORBPY20, SORWEB, SORWIF, SOVFOY, SUHBED, SULTHE01, SUTVAF, SUVJEY, SUVZEO, SUWGOG, SUXVOW, SUYWEP, SUZQIO, SUZQOU, SUZZOC, TACCAD, TACCIL, TACCUX, TAMZAK, TARLAA, TASLEE, TAWNEL, TAWSOB, TAZWOH, TCQBDX, TCQMHP, TCQNAP01, TCQPDA, TCQPEN10, TECSEZ, TEKKID, TELVIP, TENSIQ, TENSOW, TENYAO, TENYES, TERSAK, TETXUL, TEWKOV, TEXPOB10, TEZNET, TEZNIX, THLCTC, TIBZIO, TICFOA, TICREE, TIGNEE, TIJKIG, TIJTUB01, TIJVAJ, TIPFII, TIPWIY01, TIVCOS, TIVNUI, TOBSOU, TOKJUY, TOMWOJ, TOMXEA, TONGOS, TPDTCB01, TTFCAN14, TTFFAN, TUHJAH, TUPRBN01, TUQBIR, UCOTIQ, UDEKEV, UDEKIZ, UDEQUP01, UDUZEY, UDUZIC, UFETAA, UHACEM, UHACIQ, UHACUC, UHADUD, UHAFAL, UHAFEP, UHAPUO, UHAQAV, UHAQEZ, UHAQID, UHAQOJ, UHAQUP, UHEMEZ, UHEVIN, UHORUF, UHOSAM, UHOSEQ, UHOSOA, UHUNAM, UJOREQ, UKOKUA, UKOSAP01, UKOSET, UKOSIX, ULAWUZ, ULAXAG, ULAZEM, ULAZIQ, ULECEU, ULEKIG, ULEWIS, UMINAF, UMINOT, UMIQIR, UMUZAE, UNEFUO, UNEGAV, UNEZAO, UNEZES, UNIRIT, UNISUG, UNUQOK, UPOQEW, URIJEL, URIKEM, URIKUC, URILAJ, URILEN, URILUD, URISAQ, URMALN, URODEL, URODIP, UROXAM, URPRBNO1, USOBOU, UVIMES, UXOFOD, UYOTIM, UYOTUY, VABVOL, VAFWOP, VAKTOR, VAKTOS, VAKTUX, VAKVIN, VATCOI, VAWKIO, VAXQUI, VAXRAP, VAXVOH, VAXWAU, VAZBIJ, VEDFAM, VEHDIW, VEHNIF, VEMZIX, VENZOD, VEQKIL, VERJUY01, VESCUS, VEVGAG, VEVGEK, VEVGIO, VEVGOU, VEVGUA, VEVYOM, VEXQOE, VEXQUK, VEXWUQ, VIFFEV, VIFKUR, VIGFAT, VIGFEX, VIGFIB, VIGFUN, VIGGEY, VIGGOI, VIGKIF, VIHGOI, VIKLOR, VILSEP, VIPVIB, VIPXUP, VOCJAY, VOCTEO, VOCZET, VOHKUA, VORCOV, VORLOE, VUHFIO, VUQVUZ, VUZMEJ, VUZNIO, VUZZEW, VUZZOF, WABGEL, WABWEB, WADTOM, WAFFUG, WAFNOH, WAFNUN, WAJVOU, WAJWEL, WAJXAI, WANCOE, WANDEV, WANJOM, WAWDEE, WAWPAM, WEBQAX, WEDKUN, WEDLAU, WEPDIF, WEXQIA, WEYFEN, WEZXUW, WICZUF, WIFZOC, WIGCEW, WIGNIL, WINMIQ, WINTIW, WIQNIV, WIRWID, WOBJOM, WOBQIO, WOCFAX, WOCHIH, WOCHONO1, WOCVIT, WOJGUX, WOJHAE, WOJHEI, WOJHEJ, WOPPOH, WOPZUW, WOQBAF02, WOQBEJ, WOQBIN, WOQBOT, WOQBUZ, WUBYUN, WUDQAN, WURNON, WURNUT, WUVKEE, WUYROX, WUZHOP, XADVED, XAHZAH, XAMTOV, XAMWEO, XAPMAC, XAPMIK, XAPWOA, XAPWUG, XAQQEM, XAQQIQ, XAQQOW, XAQRIR, XAQVES, XAQVIW, XASCUP, XASDEA, XASDIE, XAXPIVO2, XEBFEQ, XEBFOA, XEGHEW, XEJWUF, XEJXAM, XEJXEQ, XEMCID, XEPBEB, XEPBIF, XEPBOL, XEPCAY, XEQDAA, XETLIS, XIHNOS, XIYWIM, XOBCAT, XOBCUN, XOBHIF, XODPIR, XODPOX, XOGMIR, XOGMUD, XOGNEO, XOGPAM, XOHXAU, XOJMUF, XOJNIU, XOKSUN, XOKTIC, XOLHUCO2, XOMZEG, XONBAF, XONBEJ, XONBIN, XONCIO, XONPUN, XONQIC, XOXHEY, XOZJAY, XUDCII, XUGPEU, XUNGIW, XUNGUI, XUNHIX, XUNHOD, XUNHUJ, XUNJAR, XUVPIO, XUVPUA, XUVQIP, XUVQOV, XUVQUB, XUVSAJ, XUVTEO, XUVVAM, YAFZAG01, YAGFAP, YASGAC, YASGEG, YASGIK, YASGOQ04, YATBOM, YAXWOL, YECHAQ, YEJYOB, YEPCOM, YIZXEL, YIZXIP, YOCZIB, YOCZUL, YOLJIS, YOLQOF, YUCPIW, YUPWOW, YUQHEY, YURTEL, YUWNEJ, ZAGKOH, ZAGKUN, ZAJHOH, ZAJJAV, ZAJJEZ, ZAQSOB, ZAQTUG, ZAVXAV, ZEBXOV, ZEBXUB, ZEFFEX09, ZEFVAJ, ZEVSIC, ZEVWEE, ZEXTIF, ZEXVUV, ZEXWEG, ZEXWIK, ZEZHAP, ZEZHET, ZIKNOY01, ZIKPUG01, ZIMHIO01, ZODWIY, ZOHTEX, ZOHTIB, ZOHVUP, ZOHWAW, ZOHWEA, ZOHWIE, ZOHWOK, ZOHWUQ, ZOKYON, ZUPKUQ, ZUPLAX, ZZZAKD10, ZZZGKE01, ZZZGMW02 
Table S3. Labels in the PIX database.

AJAGOH, AJAHIC, AMILEN, AMILUD01, ANOLIZ, ANOLUL, ASAXUN01, AWIHOE, BAPMAH, BICVUE01, BIFYOE, BIMSUM, BIRDIP, BIZYOA, BODSAO, BOQHOE, BORNUS, BUDWEC, CACGUK, CAXNOE, CIRNIC, CIRXAD, DITHUK, DITJAS, DITJEW, DIVVAF, DUVHOR10, DUXZAX, EBILOR, EBILUX, EFOZAB04, EXEYOW, EXIBAP, EYIXUF, FAFSEL, FOMWIN, GADGUN, GAVHER, GAVHUH01, GESCUE, GIGWOI, HIJBOR, HOFJOC, HOGGUH, HOJLIC, HOQGOL, HOQGUR, HUHQUW, IRUFIL, ITIXUE, IVOBOK, JAXSOR, JELSEY, JESMOK, JOFJUK, JUDNAX, KEWNOQ, KODXUY, LERXUD, LEWPUY, LEZQUE, LUNNAJ, LUTDUR01, MECVIB, MIRQEJ, MIZMIT, MOPYAR, NAPTOP, NEBXUP, NEPXIR, NILZOX, NPOAPL, NUHYOE, NUKWOG, NUKYAU01, OFIYOT, OFUGUR, OQOMOX, OTAZUF, PABBAV, PEFGEO, PEZQAN, PIRNOV01, PIRPEN, PIRPIR, PIRQEO, PIRQIS, PIRREP, PITYAS, PUJNIS, PYOCHP, QAFFIM, QAMQUR, QELYAI, QOGMII, QOQPOD, QUWZEO, QUWZIS, REHNAT, REQWAM, RIDFAN, RIFQAY03, RIJNEF, RIWJIQ, ROKQEN, RUXNAA, SAYNEM, SENJOK, SIDJIA, SIVBAA, SLCADC10, SOBNEE, SOLFAC, SOLFEG, SOQPAQ, SUVJEY, SUVZEO, TETXUL, TTFCAN14, TTFFAN, TUPRBN01, UHACIQ, UHADUD, UHAFEP, UMIQIR, URPRBN01, USOBOU, VAZBIJ, VIFKUR, VIPVIB, VORCOV, WABGEL, WEDKUN, WINTIW, XAPMIK, XEBFEQ, XONPUN, XONQIC, XUDCII, ZAJHOH, ZAJJEZ, ZODWIY, ZOHTEX, ZOHTIB, ZOHVUP, ZOHWAW, ZOHWEA, ZOHWIE 
Table S4. Labels in the COF database.

\begin{tabular}{|c|c|c|c|}
\hline & co-crystal & Co-former 1 & Co-former 2 \\
\hline 1. & AJAHIC & DIKPIP03 & FUMAAC01 \\
\hline 2. & AMILEN & UREAXX25 & BIPYRL04 \\
\hline 3. & ANOLIZ & PYRAZI & TARTAC01 \\
\hline 4. & ANOLUL & PYRAZI01 & TARTAL04 \\
\hline 5. & ASAXUN01 & EHOWIHO2 & PFBZAD15 \\
\hline 6. & BAPMAH & BNZQUI03 & NANILI23 \\
\hline 7. & BIFYOE & MEADEN01 & THHYDT01 \\
\hline 8. & BIMSUM & MALEHY12 & WUDVAS \\
\hline 9. & BIRDIP & BDTOLE11 & DNITBZ11 \\
\hline 10. & BODSAO & RESORA13 & UREAXX09 \\
\hline 11. & BOQHOE & PYRAZI & CLANAC \\
\hline 12. & BORNUS & BDTOLE11 & BORPUU \\
\hline 13. & BUDWEC & BENZAC99 & EHOWIHO2 \\
\hline 14. & CACGUK & EHOWIHO2 & COVJIG99 \\
\hline 15. & CAXNOE & NITPOL04 & ZZZIVG03 \\
\hline 16. & CIRNIC & JOZZIH99 & NEHQEY \\
\hline 17. & DITJEW & PCPHOL01 & CACFIW \\
\hline 18. & DUXZAX & BAPLOT02 & UREAXX09 \\
\hline 19. & EFOZAB04 & UREAXX25 & BARBAC01 \\
\hline 20. & FAFSEL & BDTOLE11 & HIPTOP \\
\hline 21. & FOMWIN & PCPHOL01 & MUBZUE \\
\hline 22. & GADGUN & PCPHOL01 & ZZZIVG03 \\
\hline 23. & GESCUE & VIDMAX02 & MALIAC13 \\
\hline 24. & HOFJOC & VOGSEP & IMAZOL13 \\
\hline 25. & HOGGUH & PICAMD05 & MALNAC16 \\
\hline 26. & HOQGOL & GLURAC13 & NIYDOO01 \\
\hline 27. & HOQGUR & ADIPACO4 & NIYDOO01 \\
\hline 28. & HUHQUW & ZZZMVY99 & VOBJEB \\
\hline 29. & IRUFIL & IRUFEH01 & IRUGAE \\
\hline 30. & JAXSOR & PYRGAL03 & TRIZIN01 \\
\hline 31. & JUDNAX & NITPOLO4 & NTPYRO11 \\
\hline 32. & KEWNOQ & ACEMID06 & ACSALA04 \\
\hline 33. & KODXUY & KODXEI & KODXOS \\
\hline 34. & LEWPUY & AMBNAC09 & AMPYRM10 \\
\hline 35. & LUNNAJ & EHOWIHO2 & CLACET07 \\
\hline 36. & LUTDUR01 & UREAXX09 & CACFIW \\
\hline 37. & NAPTOP & RESORA13 & ELOXOS \\
\hline 38. & NEBXUP & AFUYIJ01 & CLACET07 \\
\hline 39. & NILZOX & NITPOL & PYRDNO15 \\
\hline 40. & NPOAPL & NTPYRO11 & MAMPOL \\
\hline 41. & NUHYOE & UREAXX25 & IYAWAG \\
\hline 42. & NUKWOG & BENZAC99 & AMPYRM10 \\
\hline 43. & NUKYAU01 & NICOAM03 & FUMAAC01 \\
\hline 44. & OFIYOT & BEXQOK01 & ITIZUG \\
\hline 45. & OFUGUR & NITPOL04 & PYRID011 \\
\hline 46. & OTAZUF & UREAXX25 & VITBAC \\
\hline 47. & PEZQAN & CLANAC11 & NURWOM01 \\
\hline 48. & PIRNOV01 & EHOWIHO2 & IKAYEZ99 \\
\hline 49. & PIRPEN & EHOWIHO2 & WEBNAU99 \\
\hline
\end{tabular}

\begin{tabular}{|c|c|c|c|}
\hline & co-crystal & Co-former 1 & Co-former 2 \\
\hline 50. & PIRPIR & EHOWIHO2 & WEBNOI \\
\hline 51. & PIRQEO & EHOWIHO2 & DUTREQ99 \\
\hline 52. & PIRQIS & EHOWIHO2 & WEBNUO01 \\
\hline 53. & PIRREP & EHOWIHO2 & PIDGOZ01 \\
\hline 54. & PUJNIS & BENZAC99 & AMCLPY12 \\
\hline 55. & PYOCHP & PYRID011 & CHPYRD \\
\hline 56. & QAMQUR & PYRAZI01 & HYQUIN05 \\
\hline 57. & QELYAI & WUHPUK & NOCQED \\
\hline 58. & QOQPOD & PYRDNO16 & CHORLH01 \\
\hline 59. & QUWZEO & PYRAZI01 & GUFMAV \\
\hline 60. & QUWZIS & GUFMAV & HEYJOK01 \\
\hline 61. & REHNAT & HYQUIN05 & TCYNBZO1 \\
\hline 62. & REQWAM & BDTOLE11 & TCLOBQ02 \\
\hline 63. & RIDFAN & EHOWIHO2 & MNPHOL26 \\
\hline 64. & RIFQAY03 & BDTOLE11 & TCYETY03 \\
\hline 65. & RIJNEF & BDTOLE11 & BAWHEM01 \\
\hline 66. & ROKQEN & RAJGUG & MCBZAC02 \\
\hline 67. & SAYNEM & SAYNAI & CLACET07 \\
\hline 68. & SENJOK & HYQUIN05 & CUKCIU01 \\
\hline 69. & SIDJIA & UREAXX25 & ITIZUG \\
\hline 70. & SIVBAA & BNZQUI03 & BDTOLE11 \\
\hline 71. & SLCADC10 & UREAXX09 & SALIAC01 \\
\hline 72. & SOBNEE & JOZZIH99 & FURACL03 \\
\hline 73. & SOLFAC & AMBNAC09 & CYAPYR \\
\hline 74. & SOLFEG & JOZZIH99 & CYAPYR \\
\hline 75. & SUVJEY & AMPYRM10 & ТЕРНТ913 \\
\hline 76. & SUVZEO & NTPYRO11 & BIDLOP99 \\
\hline 77. & TETXUL & GIVXUE & CLACET07 \\
\hline 78. & TTFCAN14 & BDTOLE11 & TCBENQ07 \\
\hline 79. & TTFFAN & BDTOLE11 & TFBENQ \\
\hline 80. & UHACIQ & SUNKAO01 & TARTAC01 \\
\hline 81. & UHADUD & GLURAC13 & SUNKAO01 \\
\hline 82. & UHAFEP & SUNKAO01 & ZZZDUI01 \\
\hline 83. & UMIQIR & CLANAC11 & WIZFEQ01 \\
\hline 84. & USOBOU & CYAPYR & PTOLIC01 \\
\hline 85. & VIFKUR & MELAMI03 & URACIL \\
\hline 86. & VORCOV & FIXPUX & SUCACB06 \\
\hline 87. & WABGEL & BDTOLE11 & TCYNBZ01 \\
\hline 88. & WEDKUN & GLURAC13 & AMPYRZ \\
\hline 89. & XEBFEQ & BOQQUT02 & ACETAC01 \\
\hline 90. & XONPUN & BENZAC99 & XONQAU \\
\hline 91. & XONQIC & MCBZACO2 & XONQAU \\
\hline 92. & ZAJHOH & AMPYRM10 & PHTHAC01 \\
\hline 93. & ZAJJEZ & AMPYRM10 & AMBNZA99 \\
\hline 94. & ZODWIY & UREAXX25 & GLURAC13 \\
\hline 95. & ZOHVUP & UZOXEN & CLANIC06 \\
\hline 96. & ZOHWEA & UZOXEN & WEMDUN \\
\hline 97. & ZOHWIE & UZOXEN & WEMDOH \\
\hline
\end{tabular}


Table S5. Labels in the CON database.

AANHOX01, ABEGIY, ABESIK, ABEWAG, ABOHEF, ABUMIT01, ABUMOZ, ACACOX, ACANIL03, ACBNZA, ACBNZA01, ACBZPO01, ACEMID03, ACEMID06, ACENAP03, ACEQAB, ACEQUV, ACETAC01, ACETAC08, ACETPH, ACIMDC01, ACMEBZ, ACMEPT10, ACOKAE, ACOWIY, ACRDIN01, ACRDIN04, ACRLAC02, ACSALA01, ACUFIN, ACYTID, ADARAZ, ADAZUB, ADEYEO, ADIPAC03, ADIPAC08, ADIPEJ, AFEPIL, AFIGIG, AFLATM, AFLATM01, AFUYIJ, AGAWEM, AGOMIS, AHCHEX01, , AHEJEC, AHEPOS, AHGULP10, AHUZAE, AJELAC, AKOSIC, ALAGLY, AMBACO05, AMBACO06, AMBACO08, AMBACO09, AMBNAC01, AMECIE, AMECOK, AMETSL01, AMFURZ, AMGUAC02, AMIPYR, AMITEW, AMMALA, AMMPRA01, AMNTPY01, AMNTPY02, AMPYRD, AMPYRE, AMYROL01, ANCLEU, ANISAC03, ANISIC02, ANLCLA, ANLINC02, ANTCEN14, ANTQU008, APALAM, APEZEB, APHAMA, APTHOX10, APUFOH, APYFEB01, APYNAC, ARAGUV, ASALAC, ASUKAB, ASUKOP, ATCDEO, ATDZPY, ATUVIU, AVIMEY, AXALER, AXARAS, AXOSOW, AXOSOW01, AXUDED, AXUDIH, AYAYUV, AYIKUP, AYOHEB, AYOHIF, AYOHOL, AYOJED, AZASER11, AZBENC01, AZEHET, AZELAC05, AZELAC10, AZOBEN12, AZOQEM, AZOYUJ, AZOZAQ, AZSTBA, AZSTBA01, BACYEJ, BADKIC, BADKOI, BAFDIV, BAGQIJ, BAGZEQ, BAHCUI, BAHFEV, BAHNII, BAHNUU, BAHNUU01, BAHQIK, BAKFUO, BANGOM, BANGOM02, BANHOO01, BANHOO02, BANHOO03, BANMIM, BAPJEH01, BAPLOT01, BAPNUB, BAPNUC, BARBAC02, BASBAZ, BASBIH, BASBON, BASCAA, BASZEB, BASZIF, BATCEE, BAWGOW, BAWNIW, BAWNOC01, BAWPIY, BAYLAO, BAZGOY, BAZJOB01, BAZYAC, BAZYACO1, BCACEN, BCBANN, BCBANN01, BCOCAN, BCOCAN01, BDIXNA, BDTOLE10, BEBMAX, BEBMAX01, BEBXOV, BEDMON, BEFJUR, BEFTIP, BEFTIP01, BEFTOV, BEHZIX, BELLUZ, BENCLN04, BENCLN05, BENMOW07, BENZAC01, BENZDC01, BENZEN07, BENZIL02, BENZOK, BEPHOT, BEPZAX, BESSUN, BETBEH10, BETBOR, BETCEI, BEVCEK, BEYDEO01, BEYDOY, BEYZIO, BEYZIO01, BEYZIO02, BIBKUS, BIBKUSO1, BICVISBICVIS01, BICVISO2, BIFHIH, BIFXOE, BIGDUQ, BIGXAQ, BIGXEU, BIJFOP10, BIKMAJ, BINAPH11, BINDPY, BINTUN, BIPFAJ, BIPHEN04, BIPYRL04, BIPYRL04, BIRMIY, BIRYIK10, BIRYUW10, BISJIW, BISLIY, BISLOE, BISMEV, BISMEV02, BITVUV, BIVLAT, BIXGIY, BIXGIY02, BIXGIY03, BIXSOQ10, BIYMAX, BIYSEH01, BIYSEH02, BIZMED, BNORSO10, BNPHTA02, BNZQUI03, BOCCUR, BOLNAR, BOLYOQ, BOLYUW, BOMWIJO3, BONVAB, BOPXOT, BOQCUF, BOSNIG, BOXBEV, BOYMIL, BOYTUF, BOZETY01, BOZKOQ, BPHENO03, BPHENO10, BPHENO11, BPHENO12, BPHENO15, BRBNIT01, BUBLOZ, BUBNAN, BUFPOI, BUGKIX04, BUGMOG, BUGMUM, BUHGOA, BUKJOG11, BULCUG, BULVAL02, BUSFUQ, BUVBOJ10, BZAMID01, BZDIOX01, BZDMAZ01, BZMPIZ, BZOLCT, BZOXZT01, BZPTHP01, BZTROP10, BZTROP11, BZYACO, BZYACO01, CAACTY, CABTUV, CABTUV01, CACDAM, CACHAP, CADMUP, CADVEI, CADWEJ, CAHDAQ, CAJDIC, CAKPOT, CALBOG, CAMSUF, CAMWUI, CANDIN02, CAPLAC, CAPYIB, CARPOA, CARXOJ, CARZEB, CASHOT02, CATCOL13, CAVFOW, CAVGAJ, CAWDUZ, , CAWDUZ01, CAXQUO, CAYBOU, CAZFUE01, CAZFUE10, CAZGEQ, CBFBZF, CBFBZF01, CBXODL, CCACEN, CCLACN, CDBALA, CDBMPI10, CEAHOK, CECBUJ, CECGEX, CEDFAS, CEDGUP, CEFBOE, CEGDID, CEHGUT, CEHZUK, CEKGUU01, CEKREP, CEKWOE, CELKOT, CERMOB, CERMUH, CEVVOO, CEYFUI, CEZSEF, CEZVUY, CHDECL, CIBZIW, CICDIB, CICYIW, CIDJIJ, CIDTOY, CIGHEF, CIHQIT, CIHWUL, CIHWUL10, CIJVOG, CIKSEU10, CILWUP11, CIMALI, CIMETD, CIMETD01, CIMXOL, CIMXOL10, CIMXUR, CIMXUR10, CINMAC, CINMAC03, CINMAC07, CIPMUK, CIQHIU, CIQSAW, CIQSEA, CIRBEL, CIRWOR, CIRZUY, CISQIF, CIVTUW, CIVTUW01, CIWJAU, CIWMEA10, CLACET01, CLBZAC, CLBZAC, CLBZAM10, CLBZAM11, CLBZAP02, CLBZAP07, CLCYBQ, CLFORM03, CLMPYR, CLPHOL12, CLPHOL13, CLPTAZ, CLPXAD01, CLPYMD, CMALAM10, CNIXQX, COBBIE04, COBYAT, COCFOP, COCFUV, COCTCD, CODBEC, COJMUJ, COKBAF, COKBEJ, COLXUW, COLYEH, COLYIL, COLYOR, COMKAQ, COMKEU, COMXEH, COQTOR, CORNIH, CORONE02, CORZAK, COSFAR, COTMON, COUMAR02, COUMAR10, COUMAR11, COUMAR12, COVGIE, COYMOS, COYMOSO1, CPDTHO, CPTCET10, CRESOL01, CROTAC, CRYSEN01, CTCYDD, CTCYOC, CTMTNA, CUBANE, CUGBIP, CUGCOW, CUGLIA, CUGLOG, CUGPAW, CUGPEA, CUGPIE, CUGQEB, CUGQIF, CUKCAM02, CUKCAM04, CUKCIU, CUMJID, CUMJOJ, CUMZOA, CUNCAP, CUNCET, CUVDUS, CUVQAL, CUVQIT, CUVWEV, CUVYAT, CUWWEW, CUYCAA, CUZDEG, CUZGOT, CXMCYT, CYANAC, CYAPYR, CYCHEX05, CYCYPR, CYDECO, CYHCAC10, CYHCAC11, CYHEXO01, CYNGEN, CYTDEC, CYTIDI11, DABDOA, DACGOE, DACYUC, DADDER01, DADDER02, DADPAZ, DAFBIV01, DAFPUV, DAGRAF, DAHDAR, DAHFOI, DAJSIQ, DAJSIQ01, DAJVUG, DAJXIV, DAKCEX, DALBEY, DALJAC, DALNUA, DANFIH, DANFON, DANJEH, DARDIJ, DARDOP, DARSIY, DARVOH, DARVUN, DASBEF, DASBUV, DASQIX, DATMAN, DATMER, DAVHUF, DAVXED, DAWRUO, DAXCAG01, DAYBIO, DAYQEZ, DAZCOE, DAZIND, DAZNAP, DAZNAQ, DAZNAR, DAZPNE, DAZVEF, DAZVEF10, DBEZPO01, DBIBZN, DBNTHR02, DBTROP02, DBZTHP, DCACNP01, DCACON, DCBDOX10, DCCPET, DCLANT10, DCLBEN01, DCLBEN04, DCLBEN05, DCLBIP, DCLBIQ10, DCLBQN, DCLDXN04, DCLETH02, DCLNAP01, DCLNAQ, DCPYZO, DECFDP01, DEDNEF, DEDNEF01, DEFDUN, DEFDUN01, DEFFEZ, DEFYES, DEKDIG, DEKDIG01, DELCUT, DELZID, DELZOJ, DEPKIS, DEPWIE, DESKER, DESKER01, DESPUM, DESWAZ, DESWED, DESYOP, DETBAA11, DETBIO01, DETBIO10, DETFUC, DETYLE, DEWRIG, DEWVOQ, DEWVOQ01, DEXTIJ, DEZCAM01, DEZCOA, DFBPAC, DHXBZQ, DIAZNP, DIBNEH, DICCEX01, DIFMUB, DIFVET, DIFVETO1, DIKYUR, DIMNAN01, DIMPHE12, DINPEV10, DIPGIS10, DITBOX, DITHAN01, DIUREA10, DIUREA11, DIWPII, DIWPOO, DIXJIE, DIXQIK, DIXTAF, DLABUT12, DLALNI01, DLASPA11, DLGYAH, DLHIST01, DLMAND01, DLMAND02, DLMETA05, DLSERN15, DMANTL08, DMANTL09, DMANTL10, DMANTR, DMBNZA10, DMBNZA11, DMBZAC, DMBZAC01, DMCPAY, DMDHBA, DMEACL04, DMEBQU01, DMEOXA01, DMEPOL10, DMHTEZ10, DMKETD02, DMNAPH05, , DMNPTL01, DMNPYO, DMNPYO01, DMOXBA01, DMPHOL11, DMSULO, DMTPAL, DMTTDO, DMTTMC, DMTTMC03, DMURAC, DMURAC01, DMXBZA01, DMXBZQ01, DNBENZ15, DNITBZ11, DNPHOL, DODWEY, DODWIC, DOGPAQ, DOHDEJ, DOJVII, DOKXUW, DOKYAD, DOPYAI, DOSMON, DOSMUT, DOTNOP, DOTRAG, DOTREJ, DOTRUZ, DOXSOY, DOXWIW, DOXXAP, DPANTR, DPHACT07, DPIPDS, DPIPDS01, DTHDOM, DTHNPQ, DTOLTO, DTTDDO, DTTDDO01, DUBFIP, DUBHAJ, DUBHEN, DUBQAT, DUBSOJ, DUCVIH, DUDMIZ, DUFBOV10, DUFBOV11, DUFMIA, DUFQOL, DUNNEF, DUNNEG, DUNREK, DUPHEC, DURDID, DURENE05, DUSDOL, DUTRIU, DUTTAN10, DUWBEC, DUZTAT, DUZTIB, DUZYAZ, DUZYED, DZCHEP, DZTCDE, EACLID, EBENUV, EBONAK, ECEBUK, ECUXUW, EDAVUA, EDEVIT, EDMDXO, EDUGUF, EFAHAW, EFAVUC, EFAXUE, EFAYAL, EFOHIR, EFUQIG, EFUZOV, EGAKOM, EGIZEA, EHAJOM, EHEMOU, EHIXOI, EJEQUF, EKELAH, EKENUD, EKEPAL, EKUDOE, EKUDUK, ELIZAA, ELOXOS, EMIKAM, EMODEQ, EMUCOF, ENMTHA, ENVFUR, ENVFUR01, ENXBCO, ENXBCO01, EPTHOX10, EQUVER, EREQAT, ERISIH, EROREJ, ETAJUE, ETANOL, ETASIB, ETMSUL04, EVASEA, EVEVAD, EVIGEV, EVIVIP, EVIXEM, EVOSAJ, EWEYEL, EWOTUF, EWUFEH, EXEVIM, EXEWAF, EXEWEJ, EXUWAV, EYAFIT, EYIRIN, EYOHUW, EYOJAE, EZOJUZ, FACETC10, FACMOL, FACSUZ, FACTEK, FACYAJ, FAFDAS, FAFDAS01, FAFDAT, FAFDEW, FAFDIA, FAFDOG, FAHNUZ, FAHPIP, FAJYEU, FAKRIS, FALMAH, FALQAK, FARPUK, FARVIF, FASZIJ, FATVUS, FAVVIH, FAXHOBO1, FAXMAS, FAXMEW, FAXSEC, FAYGAN, FAYGAO, FBPACR, FEDGUQ, FEFBIB, FEFSAK, FEFSAK01, FEGHAA, FEGMAG, FEHVIY, FELDUW, FELRUJ, FELSEU, FEMKUD, FENCIK, FENHAH, FENZII, FEPPAR, FEPPAR01, FEPPAR02, 
FEPWAY, FEQYAC, FESNEW01, FESNIA, FEVMUO, FEVNAV01, FEVNAV02, FEWWEJ, FEXGEU01, FEYSIL, FEZJOJ, FEZJUP, FEZRAD, FIBYEW, FICVUK, FICYUM, FIHLOY, FIHLUE, FIJHIP, FIKHUC, FILHAJ, FILHAJ01, FITQUU, FITSEG, FITSIK, FITXIP, FIVGEW, FIWNUU, FIWPAC, FIWYIT, FIXDOF, FIXQEI, FIXWUE, FIYQOT, FIZJIH, FLUANA, FLUANT, FLUREN01, FOBQOD, FOGFUD, FOHPOI, FOHRIE, FOMHAZ13, FONNEC, FORIZT10, FORMAC01, FORMAM02, FOSGID, FOVJIJ, FOVYOE, FOVYOE11, FOWZAS, FOWZAS10, FPAMCA, FRONCX, FTHSEC11, FUBYOR, FUCMEV, FUDXUX, FUFBOX01, FUGJUM, FUGSOP, FUHDAN01, FUHZIS, FUHZOY, FUJFAS, FUJPOP, FULZIV, FUMAAC01, FUNSOW, FUNXIV, FUNXOB, FUPTOZ, FUTDIH, FUWPOC, FUXCUW, GACGAU, GACGEY, GACGIC, GADHAU, GADRUZ, GADXIT, GAFLEF, GAFMAB, GAGSIR01, GAGSIR05, GAKNEL, GAKNIP, GAKZIB, GALDED, GAMKUA, GANKEL, GANKIP, GANLAI, GAPWAV, GAQMER, GAQMOC, GAQWAX, GARVIE, GASWED, GAXLEW, GAXLEW01, GEBRAG, GEBSOV, GEBTUC, GECYUK, GEDHAY, GEFBAV, GEFGII, GEHMEM, GEHRIV, GEKVEY, GEKVIC, GEKXEZ01, GEKXOJ, GEKXOJ, GENFIO, GEPBOT, GEPCIN, GEPCOT, GEPCOT01, GERBEM, GERDIQ, GERZEI, GESNEZ, GESNID, GETNUO, GEVNEB, GEYTUZ, GICMIO, GICTIV, GICTIVO1, GICXEV, GIGKUC, GIHZAY, GIHZEC, GIKTUP, GIPCEN, GIPLOG, GIPPAW, GIQRAZ, GIRFAP, GISXOW, GITFIZ, GIYFIE, GIZQAH, GIZQEL, GLUCSAO3, GLUTAM02, GLUTIM, GLYALB, GLYCIN19, GLYCIN69, GLYDLA, GLYGLY22, GLYLEU10, GOCWEA, GOGPEY, GOGPIC, GOGPOI, GOGXEF, GOGXUV, GOLLUO01, GOMGEU, GOMSAC, GOMWIP, GONIOT, GOTBIA, GOVXUK, GOYLOV, GUKMUU, GULGAV, GUQLIN, GUQMIO, GUQMIO02, GUQYOG, GUSWAS, GUWCAC, GUZNUL, GUZPAT, GUZPEX, GUZPIB, HABHUN, HABMAZ, HACGAT, HADFEY, HADKAY, HAJFUT, HALKEK, HAMDAC, HAMFEH, HAMGOT, HANGIO, HASLIY, HATGUE, HAXDAL, HAXVIL, HAXVOR, HAYBIT, HAYXIO, HCCYHD, HCCYPR01, HCLBNZ11, HCLBPH, HCLHEP, HCLTRL, HCYSAC, HEBBEV, HEBYUI, HEBZOD, HECKAB, HEGHUX, HEJZAX, HEKMOZ, HEKTUM, HEKYAX01, HELVUP, HEPFUD, HEPPEX, HEPTAN02, HEPTPO, HESVUW, HESXOS, HESXUZ, HESXUZ01, HETNQU, HETNQU01, HEVHAR, HEVNUR, HEVRUV, HEXANE01, HEXCET02, HEXQAD, HEYJOK01, HEZQUY02, HIBJAD, HIBWIY, HIBWOE, HICCAX, HICXEX, HIDJEK, HIDNEN, HIDPUG, HIFNAL, HIFQUJ, HIFWOJ, HIHHAH, HIJCAE, HIJCEI, HIKZAC, HILWEF, HIMMAL, HIRBIV, HIRSUX, HIWSAH, HIXJEE, HIYNUY, HMPYTH, HOFGEO, HOFHEQ, HOMSOR, HOMSUX, HOMYAJ, HOPJUR, HOPXOZ, HOQBIY, HOQPIO, HOSNEI, HOSNIM, HOVXOF, HOYCOO, HOYCUU, HPTCHL, HTIEPO, HUCTIJ, HUDZAI, HUFZIS, HUKROU, HULSEM, HUMJAA, HUMTEP01, HUQXEW, HUQXOG, HURJEK, HUSXAU, HUWGUB, HUZJUH, HXMPOL, HXMTAM23, HXPHIM, HYFURA, HYFURC, HYXBUR10, IBETEO, IBUHES, IBUHIW, IBUHOC, IBUHUI, IBURAM, IBURAM, ICACEN, ICRFRA10, ICRFRB10, IDEPEN, IDOQUO, IDUWEJ, IETPYA10, IFAGAY, IFILEO, IFIMIT, IHECIH, IHEMAJ01, IHEMIR, IHEMIR01, IJIVEC, IJUHUQ, IJUJAY, IKIDAH, IMAZOL13, IMDACC01, IMEMUI, IMIJUJ, IMIPOJ, IMITED, IMITIH, IMIVOP, INDBUA01, INDBUA02, INDDON, INDPRA01, INERAU, IOBNITO1, IPEPPL, IPEXEH, IPMEPL, IQAREY, IQILID, IROMUY, IRONAF, IROREM, IRUYIE, ISADIP, ISAJAN, ISAJIV, ISALAP, ISATINO4, ISIGUM, ISIRINO1, ISODIE, ISPIMP, ISPIMP01, ISUQUI, ISURAP, ITOSIU, IVAGUI, IVASIH, IWILEG, IWIPOU, IWODIH, IWODON, IWUCAF, IWUFAH, IXEGEX, IYAWAG, IYIMIM, IYIMUY, IYINAF, IYINAF01, IZAMAX, IZAWUB, IZAXAI, IZECUL, IZEDUM, JABPUY, JABQAF, JABXAM, JACCIZ, JACTUC, JAHZIB10, JAKGIL, JAKKIP, JAMHOU, JAPBEI, JAPVAX, JAQDAH, JAQGIS, JARDEM, JARQUP01, JASGIT, JAYDUI, JAYNOM, JAZNII, JAZQAC, JEFMUD, JEMGIS, JESJEX, JEVNEE, JEXNAB, JINZAI, JIPFAQ, JIPNUR, JISJAX, JITMEE, JIXWIW, JIYVUI, JOFKOE, JOLGUM, JOLGUM10, JOPFAV, JOTGII, JOTKUY, JOWWIB, JOXBED, JOZVEZ, JUBJAR, JUBKIA, JUBLAT, JUCDOA, JUDZEN, JUDZIR, JUFBAN, JUFBUH, JUFNED, JUFNIH, JUJZAP, JUJZIX, JUKLUW, JUKMAD, JULREN, JUNCUQ, JUNFUT, JUQCUT, JUTXUR, JUXHUF, JUZDEN, JUZDIR, KABJON, KABLOO, KAFBID, KAGBID, KAHROA10, KAHRUG, KAJRET, KAQPOH, KAQPUN, KASJI, KATPIG, KAXMAY, KAXWOW, KAXXAI, KEFYOK, KEKWOM, KEKYEE, KEMPOH, KEMTOM, KEMTUS, KEQXIO, KERXUB, KERYAI, KERZUD, KETVEK01, KEYNOR10, KEZNIN, KIDCOP, KIDCUV, KIGCAE, KIMDEP, KINWIN, KIWVIW, KIXSUF, KOBSID, KOBXOO, KODXAD, KOJGUM, KOJZEO, KONDEW, KONJOM, KORZIA, KOTDUS, KOVHAE, KOXBOO, KUFVAI, KUNXEW, KUSVUP, KUTGEL, KUTHAI, LABNIL, LABQUA, LACFEB, LAGWOH, LAGWOH01, LAKDEH, LAKJEM, LAKJOW, LALNIN23, LALXAX, LANJER, LANPOG, LANXON, LAQFIT, LASPRT, LAXZAM, LCYSTN25, LEBMIP, LECFUV, LECFUV, LEDZEZ, LEHBAC, LEHQIZ, LEHQUL, LEJMAP, LEJTOK, LELXAB, LEQVUYO1, LEVYAM, LEVYOB, LEWWUG, LEZHUT, LEZJAB, LEZJAB01, LGLUAC11, LHISTD13, LIHFIR, LIHFOX, LIHTOL, LIJPOJ, LIJQAW, LIKBOW, LIKBOW01, LIKCEN, LIMKIB, LIQVOW, LISVIT, LIWGOO, LIWQAJ, LIWXEW, LOCHIV, LOCPUQ, LOCTUS, LOCVIJ, LODMUM, LOFSEE, LOMHOK, LORNOW, LOSBIEO1, LSERIN27, LTHREO03, LUHCAS, LUNQAM, LUNQEQ, LUNQOA, LUNQUG, LUPWAU, LURZAZ, LUVSIF, LUVSOL, LVALIN01, MACLAE, MADENC10, MAFXAT, MAFXEX, MALEHY01, MALEHY11, MALEHY12, MALIAC11, MALNAC06, MALOAM, MALONT, MANTHR03, MARBUD, MASBUD, MATDEQ, MAVRAD, MAWLAX, MAYXEP, MAYXIT, MCBZAC, MCCARD, MCRZMB, MDTHAC, MEADENO1, MEBNUD, MEBPIT, MEBPOZ, MECHLF12, MEHAMC01, MEHPAR, MELAMI04, MEPSUW, MEPTAD, MESITN, METNAM04, METOLS10, METXOZ, MEUREA, MEXCUP, MFZCHZ, MHCYTC, MIKTEG, MINCOB, MIQUINO1, MIVVOC, MIXPEO, MIYDED, MIYJIN, MLEICA, MNBZAC04, MNDXTO, MNIANL02, MNTDMA01, MOFNIE01, MOFQOO, MOHCES, MOLHAX, MOLHIF, MOMKII, MOPJUW, MOPRDA10, MOPRDB10, MOQCAW, MOQCEA, MORPEO, MOZZOR, MPSUFO01, MPSUFO01, MPTRZS, MPTRZS01, MPYAZO10, MPYAZO11, MPYRAZ02, MQAZOX, MTFULC, MTHPHE01, MTNANL, MTROLA10, MTZOPZ10, MUBZOY, MUBZUE, MUCPIK, MUHFOK, MUKMAG, MUKVOD, MUKVUJ, MUMQIV, MUMQOB, MURPIY, MURPOE, MUSVUR, NABRAK, NABREO, NACVAL, NACXEU, NADCOL, NAGVUM, NAGWAT, NAHFIL, NAHMUE, NAHNIT, NAHPIV, NAKMUH, NANDIP, NANDUB, NANILIO2, NAPHACO2, NAPHOL, NAPHQU, NAPHQU01, NAPHTA10, NAPOAC01, NAPTAN02, NAPTYR, NAQRIG, NAQRIG01, NATZCX, NAVPAD, NAYJED, NAYNUX, NAYZOD, NAZZAP01, NAZZAP02, NBONAN01, NBORAN02, NBORAN10, NBORAN11, NBZOAC02, NBZOAC04, NECNAK, NEDMOY, NEJRUQ, NEKREA, NEKZEI, NEMLEW, NENJOF, NENTUV, NEPGCLO3, NEQBUI, NEQRIM, NEQSEJ, NETJUR, NETROT, NEXMOU, NEZNEL, NFMLEB, NICOAC02, NIFCEK, NIFHOA, NIKCUG, NIKVUZ, NILKOI, NIMDUI, NIMFAQ, NIMFOE01, NIMFOE02, NINHYD02, NINZUF, NITBAL02, NITOLU, NITPOL02, NITPOL03, NITPOL08, NITSOY, NITSUE, NIVPAJ, NIVPAJ01, NIVRIU, NIXQIU, NMALAM, NMCABZ, NMEFUL, NMLALA10, NMYPHD, NMZNON, NMZNON01, NOCPEA, NOCPIE, NODTIJ, NOETNA, NOETNA02, NOGUNA01, NOKBOE, NOKKUU, NOKWIT, NOPFIH, NOREPH01, NOTSAQ01, NOYGIR, NOZXEF, NSMACM, NTRGUA03, NTSALA, NUJDIG, NUNKEM, NUQPIZ, NUQXEC, NUQXEC01, NURVUS, NURWOM03, NUSGIR, NUTTIF, NUTXIJ, NUYPEC, NXENAM, NXENAM01, OBEYEB, OBIBAC, OBIZEE, OCASER, OCDBDO10, OCEBAA, OCHTET, OCHTET03, OCHTET12, OCTANE01, OCTPIN10, ODENUH, ODETOH, ODIKES, OFEQUM, OFNAPH01, OGETEA, OGOGUM, OGOHAT, OIBZAC01, OKIFOE, OKISAC, OKISEG, OKOXOC, OKOXUI, OKOYET, OLAWUT, OLOBIA, ONITPH, OPADNA, OPADNB, OPDDTE, OPOXAZ, OPOXAZ01, OPTCET, OSOLIS, OSOLOY, OVERAT01, OXALAC04, OXALAC06, OXTZOL01, PABKUY, PABZOJ, PABZUP, PAHZII, PAHZOO, PAKPAS, PALDOW, PANQUO, PANQUO01, PAPHOD01, PAPWUY, PARBAC11, PARJIB, PAVZOD, PAVZUJ, PCHSAN, PCHSAN01, PCLCHX10, PDHTEZ, PDHTEZ01, PEDGAI, PEDZEE, PENRAC, PEQXOY, PERLEN04, PERYTN01, PETNEI, PETNER, PEVNUZ01, PEVRAJ, PEWXAQ01, PEWXAQ02, PFBZAC, PFBZAD10, PHBALD11, PHBENZ, 
PHENAN08, PHENAT02, PHENAZ02, PHENAZ02, PHENAZ10, PHENAZ11, PHENSS01, PHTETZ01, PHTHAO, PHYDZC01, PICRAC, PIDGOZ, PIGVIM, PIKGEV01, PIMBAP, PIMELA05, PIMELA06, PIMTAZ, PIMTAZ01, PINPAD, PINPAD01, PIPDCL24, PIRPEL, PIRPEL, PIRZIA, PIWXIK, PLACTA01, PLBULC, PLBULD, PNCLBZ, POBDEP, POGNUU, POKKAB, POPGEG, POPGEH, PORWOI, POVKEQ, POWBOS, POXHYN10, POXHYN11, PRMDIN01, PTCDEC, PTCDEC01, PTCHLD, PTOLIC, PTOXEC, PUBGIC, PUFFOL01, PUNYIG, PUNYOM, PUPBAD, PUPBAD01, PUPBAD02, PUPZEF, PUVVUX, PUYHOG, PUYQUV, PVVAWA01, PYDMIM, PYDZHC, PYMDAN, PYMSUL10, PYRAZI01, PYRENE02, PYRZIN01, PYRZIN02, PYRZOL02, PYRZOL04, PYZPYT01, PYZPYT10, QACRIV01, QADVIA, QAKCOU, QALQEA01, QALQIE, QAMSUS, QAMVEG, QANRUT, QATTOU, QAXMEH, QAXMEH03, QAXMEH05, QECHOV02, QEDKOB, QEHVEF, QEKDAL, QEKQIG, QEKQUS, QEKYAG, QEPNUW, QERCEW, QESGOL01, QESKUV, QETKOP, QEXNIR, QEYGAD, QEYGIL, QICVAZ, QIKDET, QIMGAU, QIMHID, QIPTUE, QIWJAI, QIXCEG, QIYQUK, QIZHIQ, QIZHIQ01, QOCNAX, QOCNAX01, QOYJOD, QOYNOH, QOYNOH01, QQQBTY01, QQQCIV04, QQQCIV05, QQQCIV08, QQQFED02, QQQFNG03, QQQFUS01, QUCMAD, QUDFUQ, QUINAZ, QULNIV, RAFXOM, RAHDUZ, RAHFAH, RAKZUY, RAMVOQ01, RAPWIP, RAQHAS, RAQHEW, RARLON, RAVNOR, RCLOTH, RDCPLB01, REGYEH, REHCAK, REKJUN, REQQAG, REQYER10, RESLEG, RETMEJ, RETYAR, REZNUG, RIBVIH, RIGZAJ, RIHJIB, RIHMAX, RIKXIS, RIYFAG, RIZYEE, RMNALA10, ROFGUO, ROLTOB, ROPPAN, ROPPER, RUHCUS01, RUVQAA, RUXXEN, SACHEK, SACPAN, SADHUA, SAFFEK01, SAFPIY, SAFPIY01, SAFWAZ, SAKFUG, SALIAC16, SAMDUG, SAPZEO, SARBUI, SARPOR, SATNEH, SAWJIJ, SAWJIJ10, SAWZIA, SAZXEW, SDPPCX, SEDTUQ06, SEDTUQ09, SEFDIQ, SEGCAI, SEGHUH, SEGWIK, SEJGAR, SEMHOI, SEMYIT, SENREJ, SETHEE, SEZCUV, SEZFUZ, SEZMEP, SIBJIX, SIDFIU, SIFGIX, SIFLOI, SIFLOI01, SIGYEN, SIKLIH07, SIKQOS, SIMRAH, SINYUK, SIQZAT, SISNOX, SISPAL, SITFOR, SIVHOU, SOBCER, SOBFEU, SOBFOE, SOBNED, SOGJUU, SOLJEJ, SOXGES, SOYMOI, SUCACB11, SUCANH, SULAMD02, SULAMD05, SULAMD06, SULZUV, SUQFITO0, SUQFIT01, SURCEN, SURCIR, SUSCOY, TACQUJ, TAFLAO, TAHFUD, TAHPIB, TALIZOO, TALIZO1, TAMCRT, TAMFET01, TARGID, TARPAF, TARTAC, TARTAL, TARTAL01, TARTAL02, TATNBZ, TAURIN07, TAYWOG, TAYYIB, TAYYOH, TAZCAZ, TAZTAZ, TBTYAC00, TBTYAC01, TCBENQ01, TCBENQ07, TCBENZ, TCBENZ03, TCCYHX10, TCHLBZ, TCHLBZ03, TCLBEN02, TCLBZN, TCMSHD, TCYCPR, TCYETY03, TCYMET, TCYNBZO1, TCYNEO, TCYQME, TCYQME02, TEDQAU, TEFNUN, TEJPIH, TEKKAV, TEKWOV, TELPIJ, TELPOP, TELYAK01, TEMBES01, TEMWUD, TEPHTH00, TEPHTH01, TEPHTH12, TEPNIT04, TEPNIT06, TEPNIT06, TEPNIT11, TERMIN, TETDAM06, TETRAZ02, TETZOL00, TETZOL02, TEVHEH, TEZBIJ, TEZPDZ10, TFACET, THACEM01, THALID03, THALID10, THCYTO10, THIANT03, THPYDO, THTFBD, TICHOC, TICHOC01, TICVUW, TICXUY01, TICZOU, TIHGOH, TIJLIH, TIPVOD, TITNEP, TITNER, TIZMAQ, TMBZAC01, TMBZAC05, TMEBZA, TMETTS01, TMETTS02, TMETTS21, TNAPHB, TNAPHC, TNBENZ10, TNBENZ13, TNIOAN, TNOXYL, TNPHNT, TOBBIV, TOBRUX00, TOBRUX01, TOFNAD, TOHTUG, TOLETY, TOLUEN, TORTEA, TOVMAS, TOXOCN, TOYQAZ, TOYWAF, TOYWEJ, TOYWIN, TPENAC02, TRAZOL03, TRIPHE11, TRITAN10, TRIZIN01, TROXAN, TSEMHC, TSTCDO, TSTILB05, TTMCDN, TUBYIY01, TUBYIY03, TUKQUM, TUKWOL01, TULSEY, TURCIL02, TUSQON, TUXLUT, TUXMAA, UBEREY, UBIMIB, UCAWOL, UCECAG03, UCEROK, UDAHOW, UDOFUP, UFEBIQ, UFESOP, UFILUQ01, UFOBIA, UFOBOG, UFUXOI, UGUHAF, UHACIP, UHAMAR, UJOHAC, UJOHEG, UJUKEP, ULEJEB, ULIMEH, ULOKUB, UNECAS, UPIDED, URAZIX, UREAXXO9, USAJAA, UWETOG, UXAZID, UXEWOK, UXOQII, UXOQOO, UYIBIO, VABROF, VABVAV, VABVEZ, VACRAT, VACWAZ, VACWED, VAHFIU, VALIDLO3, VANLAX, VANYEO, VAPREJ, VAQREM, VARZOD, VASDOI, VATKEH, VATPAI, VAVTOB, VAVVET, VAYIO, VAZVEY, VEBTOM, VEBTOM, VECVEF, VECXIL, VECXUY, VEDCEM, VEGHEU, VEJDIX, VEJPUV, VEJRAD, VEKQUX, VEKSIN, VESBEB, VESBOL, VESBUR, VESDAZ, VESROB, VETWOH, VETWUN, VEVCEF, VEXNIW, VEXNOC, VEXPUJ, VEXXUR, VEYPEV, VICREE, VIDQAB, VIDSIK, VIFBER, VIFGIA00, VIFGIA01, VIGLAA, VIKKEG, VIKNAE, VINZIC, VIRGOS, VIWCOT, VOBJAX, VOBJEB, VOBNAB00, VOBNAB01, VOBVEN, VODWEQ, VOFBUN, VOGSEP, VOHJEH, VOHNAH, VOLBED, VOLXOK, VONZON, VOPYII, VORNIZ, VORVUT, VORWAA, VUBZUN, VUGMAM, VUGMEQ, VUGSIZ10, VUGWAV, VUYGEB, WACYAB, WAFYOR, WAJXUB, WAKBIU, WAKDIX, WAKHEW, WAKSUX, WAMDEU, WATFAZ, WAVKIN, WECXAE, WEDJEV, WEFQII, WEGTOR, WEGXEL, WEJHUO, WEJRIM, WEMFAV, WENZOF, WESBEB, WESBIF, WESBOL, WEWPUJ, WEZCAG, WIFKEB, WIGYIV, WIHDEY, WIKCUO, WIQDIJO1, WIYBIP, WIZFAM, WIZFEQ, WOBGIE, WOBLUU, WOKWAV, WOSTUT, WOSVAB, WOSWAC, WUQKUP, WUQLAW, WURWIP, WUSPAB, WUVTUC, XADPAU, XADPEY, XAFTUV, XAFVUX, XAJYOX, XAKZIS, XALTUZ, XANYOB, XAQFAX, XAQNOT, XATJUX, XATMOV, XATQUEO2, XAVQOB, XAYMEP, XAYMIT, XAYSIZ, XAZZON, XAZZUT, XEBKUL, XECDUF01, XEDDAM, XEDTOP, XEGHOG, XEJNUV, XEJNUV01, XEQJOS, XETJIQ, XEXXED, XICCUI, XIGMAC, XIHMEG, XIHMIK, XIPWID, XIQTEX, XISWEC, XISXED, XIXQOL, XOCJEEO0, XOCJEE01, XODGIG, XOFCUQ, XOHCOM, XOHCUS, XOHHEI, XOKWID01, XUDWEY, XUDWOI, XUGLEQ, XULDUD00, XULDUD01, XUMWEH, XUMWIL, XUPFUJ, XUPGAQ, XUQLUQ, XYANAC, YACGAK, YACGAK01, YAHCAN, YAJQUW, YAMHID, YAMHID03, YANCEW, YANCIA, YAVCON, YAVJOU, YAVPIU, YAWNIT, YAXQEV, YAZBUX, YEDKEX, YEGCAP, YEGFIZ, YEGFOF, YEGHIB, YEGTIO, YEKQAG, YELSEO, YELZIZ, YELZOF, YEPFEE, YERJIO, YERJOU, YEXQUN, YEYWOP, YEZJOD, YICFAR, YIDCOD, YIDCUJ, YIDLECOO, YIDLEC01, YIFDUN, YIJHOO, YIPZUT, YIRCIM, YOBQEL, YODLIN, YOHHIN, YOLBOQ, YOLDAF01, YOLNIW, YOMMAP, YOSHOD10, YOWXIR, YOXGIB, YUBTOE, YUHFEM, YUHFOW, YUNLUO, YUXPEN01, YUYHIJ00, YUYHIJ02, YUYLAF, YUYMOU, YUYPUD, YUYPUD01, YUYPUD02, ZATVOF, ZBCNON01, ZECWOV, ZEDPIH, ZEDPIH01, ZEGCUJ, ZELDOJ00, ZELDOJ01, ZELKUW, ZEQVIC, ZERLEP, ZEXQAU, ZIDCEU, ZIDCIY, ZIJLUB, ZIKWAR, ZILHOR01, ZILHOR02, ZIPXOL, ZIWDOA, ZIYMOJ, ZIZFET, ZOFSAO, ZOKRUM, ZOLWEC, ZOLWIG, ZOSNUQ, ZOSPOM, ZOYLII, ZOYLUU, ZOZZUJ01, ZUHDUB, ZUHFAJ, ZULDOZ, ZUMKEX, ZZZAOS02, ZZZBFP01, ZZZBLP02, ZZZBLS01, ZZZDUI01, ZZZEKW02, ZZZFYW01, ZZZGVU02, ZZZIIO01, ZZZITY01, ZZZIYE01, ZZZIZA01, ZZZJIQ02, ZZZKAO01, ZZZKAY01, ZZZKPE01, ZZZKPE03, ZZZLYS01, ZZZMKS01, ZZZMLY01, ZZZMUC01, ZZZNQSO3, ZZZPRC02, ZZZQSC01, , ZZZQSC02, ZZZSPE01, ZZZWGK01 
Table S6. Correspondence between the PIX co-crystals and their COF Co-formers, with energies in $\mathrm{kJ} \cdot \mathrm{mol}^{-1}$. Repeated entries refer to combinations involving structures determined at different $\mathrm{T}$, or in different polymorphic forms.

\begin{tabular}{|c|c|c|c|c|c|}
\hline co-crystal & Co-former 1 & Co-former 2 & Et(PIX) & $\mathrm{Et}(\mathrm{PIX})_{1}$ & $\mathrm{Et}(\mathrm{PIX})_{2}$ \\
\hline AJAHIC & DIKPIPO3 & FUMAAC01 & -121.0 & -125.2 & -124.1 \\
\hline AMILEN & UREAXX09 & BIPYRL04 & -104.9 & -104.2 & -89.5 \\
\hline AMILEN & UREAXX25 & BIPYRL04 & -104.9 & -105.6 & -89.5 \\
\hline ANOLIZ & PYRAZI & TARTAC01 & -117.7 & -59.5 & -167.6 \\
\hline ANOLIZ & PYRAZI01 & TARTAC01 & -117.7 & -63.6 & -167.6 \\
\hline ANOLIZ & PYRAZI & TARTAL04 & -117.7 & -59.5 & -165.2 \\
\hline ANOLIZ & PYRAZI01 & TARTALO4 & -117.7 & -63.6 & -165.2 \\
\hline ANOLIZ & PYRAZI & TARTAM & -117.7 & -59.5 & -151.9 \\
\hline ANOLIZ & PYRAZI01 & TARTAM & -117.7 & -63.6 & -151.9 \\
\hline ANOLIZ & PYRAZI & ZZZDUI01 & -117.7 & -59.5 & -166.3 \\
\hline ANOLIZ & PYRAZI01 & ZZZDUI01 & -117.7 & -63.6 & -166.3 \\
\hline ANOLUL & PYRAZI & TARTAC01 & -113.0 & -59.5 & -167.6 \\
\hline ANOLUL & PYRAZI01 & TARTAC01 & -113.0 & -63.6 & -167.6 \\
\hline ANOLUL & PYRAZI & TARTALO4 & -113.0 & -59.5 & -165.2 \\
\hline ANOLUL & PYRAZI01 & TARTALO4 & -113.0 & -63.6 & -165.2 \\
\hline ANOLUL & PYRAZI & TARTAM & -113.0 & -59.5 & -151.9 \\
\hline ANOLUL & PYRAZI01 & TARTAM & -113.0 & -63.6 & -151.9 \\
\hline ANOLUL & PYRAZI & ZZZDUI01 & -113.0 & -59.5 & -166.3 \\
\hline ANOLUL & PYRAZI01 & ZZZDUI01 & -113.0 & -63.6 & -166.3 \\
\hline ASAXUN01 & EHOWIH01 & PFBZAD15 & -105.9 & -112.0 & -79.7 \\
\hline ASAXUN01 & EHOWIHO2 & PFBZAD15 & -105.9 & -112.8 & -79.7 \\
\hline BAPMAH & BNZQUI03 & NANILI23 & -86.0 & -60.9 & -100.5 \\
\hline BIFYOE & MEADEN01 & THHYDT01 & -121.9 & -135.2 & -106.5 \\
\hline BIFYOE & MEADEN01 & THHYDT02 & -121.9 & -135.2 & -114.7 \\
\hline BIFYOE & MEADEN02 & THHYDT01 & -121.9 & -135.5 & -106.5 \\
\hline BIFYOE & MEADEN02 & THHYDTO2 & -121.9 & -135.5 & -114.7 \\
\hline BIFYOE & MEADEN05 & THHYDT01 & -121.9 & -137.3 & -106.5 \\
\hline BIFYOE & MEADEN05 & THHYDT02 & -121.9 & -137.3 & -114.7 \\
\hline BIMSUM & MALEHY01 & WUDVAS & -111.0 & -111.2 & -96.9 \\
\hline BIMSUM & MALEHY11 & WUDVAS & -111.0 & -116.2 & -96.9 \\
\hline BIMSUM & MALEHY12 & WUDVAS & -111.0 & -111.3 & -96.9 \\
\hline BIRDIP & BDTOLE11 & DNITBZ11 & -86.1 & -83.1 & -84.4 \\
\hline BODSAO & RESORA13 & UREAXX09 & -104.6 & -104.9 & -104.2 \\
\hline BODSAO & RESORA13 & UREAXX25 & -104.6 & -104.9 & -105.6 \\
\hline BOQHOE & PYRAZI & CLANAC & -94.8 & -59.5 & -125.5 \\
\hline BOQHOE & PYRAZI & CLANAC11 & -94.8 & -59.5 & -130.1 \\
\hline BOQHOE & PYRAZI01 & CLANAC & -94.8 & -63.6 & -125.5 \\
\hline BOQHOE & PYRAZI01 & CLANAC11 & -94.8 & -63.6 & -130.1 \\
\hline BORNUS & BDTOLE11 & BORPUU & -95.7 & -83.1 & -97.4 \\
\hline BUDWEC & BENZAC99 & EHOWIHO1 & -103.6 & -84.8 & -112.0 \\
\hline BUDWEC & BENZAC99 & EHOWIHO2 & -103.6 & -84.8 & -112.8 \\
\hline CACGUK & EHOWIH01 & COVJIG99 & -104.3 & -112.0 & -88.0 \\
\hline CACGUK & EHOWIHO2 & COVJIG99 & -104.3 & -112.8 & -88.0 \\
\hline CAXNOE & NITPOL & ZZZIVG03 & -85.6 & -91.3 & -70.5 \\
\hline CAXNOE & NITPOLO4 & ZZZIVG03 & -85.6 & -95.1 & -70.5 \\
\hline CIRNIC & JOZZIH99 & NEHQEY & -104.7 & -122.6 & -78.0 \\
\hline CIRXAD & KUTHAI & SUCACB06 & -133.2 & -100.6 & -117.7 \\
\hline DITJEW & PCPHOL01 & CACFIW & -109.9 & -129.4 & -57.9 \\
\hline DUXZAX & BAPLOT01 & UREAXX09 & -119.8 & -128.9 & -104.2 \\
\hline DUXZAX & BAPLOT02 & UREAXX09 & -119.8 & -123.9 & -104.2 \\
\hline DUXZAX & BAPLOT01 & UREAXX25 & -119.8 & -128.9 & -105.6 \\
\hline DUXZAX & BAPLOT02 & UREAXX25 & -119.8 & -123.9 & -105.6 \\
\hline EFOZAB04 & UREAXX09 & BARBAC01 & -110.4 & -104.2 & -108.0 \\
\hline EFOZAB04 & UREAXX09 & BARBAC02 & -110.4 & -104.2 & -110.2 \\
\hline EFOZAB04 & UREAXX25 & BARBAC01 & -110.4 & -105.6 & -108.0 \\
\hline EFOZAB04 & UREAXX25 & BARBAC02 & -110.4 & -105.6 & -110.2 \\
\hline FAFSEL & BDTOLE11 & HIPTOP & -88.2 & -83.1 & -82.1 \\
\hline FOMWIN & PCPHOLO1 & MUBZUE & -106.2 & -129.4 & -70.6 \\
\hline GADGUN & PCPHOL01 & ZZZIVG03 & -99.9 & -129.4 & -70.5 \\
\hline GESCUE & VIDMAX & MALIAC12 & -119.9 & -127.3 & -108.3 \\
\hline GESCUE & VIDMAX02 & MALIAC13 & -119.9 & -118.5 & -108.5 \\
\hline HOFJOC & VOGSEP & IMAZOL13 & -108.9 & -120.0 & -88.0 \\
\hline HOGGUH & PICAMD04 & MALNAC16 & -99.2 & -102.7 & -102.8 \\
\hline HOGGUH & PICAMD05 & MALNAC16 & -99.2 & -93.4 & -102.8 \\
\hline HOQGOL & GLURAC13 & NIYDOO01 & -124.2 & -128.6 & -114.8 \\
\hline co-crystal & Co-former 1 & Co-former 2 & Et(PIX) & $\mathrm{Et}(\mathrm{PIX})_{1}$ & $\mathrm{Et}(\mathrm{PIX})_{2}$ \\
\hline HOQGUR & ADIPAC04 & NIYDOO01 & -130.0 & -126.9 & -114.8 \\
\hline HOQGUR & ADIPAC07 & NIYDOO01 & -130.0 & -120.4 & -114.8 \\
\hline
\end{tabular}

\begin{tabular}{|c|c|c|c|c|c|}
\hline co-crystal & Co-former 1 & Co-former 2 & Et(PIX) & $\mathrm{Et}(\mathrm{PIX})_{1}$ & $E t(P I X)_{2}$ \\
\hline HOQGUR & ADIPAC08 & NIYDOO01 & -130.0 & -132.6 & -114.8 \\
\hline HOQGUR & ADIPAC11 & NIYDOO01 & -130.0 & -125.5 & -114.8 \\
\hline HOQGUR & ADIPAC13 & NIYDOO01 & -130.0 & -126.4 & -114.8 \\
\hline HOQGUR & ADIPAC15 & NIYDOO01 & -130.0 & -128.8 & -114.8 \\
\hline HOQGUR & ADIPAC17 & NIYDOO01 & -130.0 & -125.7 & -114.8 \\
\hline HOQGUR & ADIPAC20 & NIYDOO01 & -130.0 & -127.2 & -114.8 \\
\hline HUHQUW & ZZZMVY99 & VOBJEB & -93.3 & -114.3 & -71.5 \\
\hline IRUFIL & IRUFEH & IRUGAE & -115.3 & -87.3 & -140.4 \\
\hline IRUFIL & IRUFEH01 & IRUGAE & -115.3 & -88.5 & -140.4 \\
\hline JAXSOR & PYRGAL03 & TRIZIN01 & -94.8 & -122.4 & -59.8 \\
\hline JUDNAX & NITPOL & NTPYRO11 & -91.2 & -91.3 & -83.2 \\
\hline JUDNAX & NITPOLO4 & NTPYRO11 & -91.2 & -95.1 & -83.2 \\
\hline KEWNOQ & ACEMID01 & ACSALA04 & -95.0 & -75.7 & -113.7 \\
\hline KEWNOQ & ACEMID02 & ACSALA04 & -95.0 & -76.4 & -113.7 \\
\hline KEWNOQ & ACEMID06 & ACSALA04 & -95.0 & -80.4 & -113.7 \\
\hline KODXUY & KODXEI & KODXOS & -95.4 & -88.9 & -93.4 \\
\hline LEWPUY & AMBNAC04 & AMPYRM10 & -107.9 & -117.0 & -94.5 \\
\hline LEWPUY & AMBNAC06 & AMPYRM10 & -107.9 & -104.4 & -94.5 \\
\hline LEWPUY & AMBNAC09 & AMPYRM10 & -107.9 & -106.5 & -94.5 \\
\hline LUNNAJ & EHOWIHO1 & CLACET07 & -98.6 & -112.0 & -85.0 \\
\hline LUNNAJ & EHOWIHO2 & CLACET07 & -98.6 & -112.8 & -85.0 \\
\hline LUTDUR01 & UREAXX09 & CACFIW & -95.3 & -104.2 & -57.9 \\
\hline LUTDUR01 & UREAXX25 & CACFIW & -95.3 & -105.6 & -57.9 \\
\hline NAPTOP & RESORA13 & ELOXOS & -103.6 & -104.9 & -99.9 \\
\hline NEBXUP & AFUYIJO1 & CLACET07 & -115.0 & -115.2 & -85.0 \\
\hline NILZOX & NITPOL & PYRDNO13 & -89.2 & -91.3 & -76.0 \\
\hline NILZOX & NITPOL04 & PYRDNO13 & -89.2 & -95.1 & -76.0 \\
\hline NILZOX & NITPOL & PYRDNO14 & -89.2 & -91.3 & -81.1 \\
\hline NILZOX & NITPOLO4 & PYRDNO14 & -89.2 & -95.1 & -81.1 \\
\hline NILZOX & NITPOL & PYRDNO15 & -89.2 & -91.3 & -80.8 \\
\hline NILZOX & NITPOL04 & PYRDNO15 & -89.2 & -95.1 & -80.8 \\
\hline NILZOX & NITPOL & PYRDNO16 & -89.2 & -91.3 & -79.4 \\
\hline NILZOX & NITPOL04 & PYRDNO16 & -89.2 & -95.1 & -79.4 \\
\hline NPOAPL & NTPYRO11 & MAMPOL & -94.6 & -83.2 & -107.7 \\
\hline NUHYOE & UREAXX09 & IYAWAG & -129.0 & -104.2 & -153.4 \\
\hline NUHYOE & UREAXX25 & IYAWAG & -129.0 & -105.6 & -153.4 \\
\hline NUKWOG & BENZAC99 & AMPYRM10 & -94.8 & -84.8 & -94.5 \\
\hline NUKYAU01 & NICOAM03 & FUMAAC01 & -116.6 & -113.3 & -124.1 \\
\hline OFIYOT & ВЕХООК01 & ITIZUG & -101.5 & -123.0 & -64.7 \\
\hline OFUGUR & NITPOL & PYRIDO11 & -94.5 & -91.3 & -93.3 \\
\hline OFUGUR & NITPOL04 & PYRIDO11 & -94.5 & -95.1 & -93.3 \\
\hline OTAZUF & UREAXX09 & VITBAC & -116.5 & -104.2 & -113.6 \\
\hline OTAZUF & UREAXX25 & VITBAC & -116.5 & -105.6 & -113.6 \\
\hline PEZQAN & CLANAC & NURWOM01 & -114.2 & -125.5 & -67.9 \\
\hline PEZQAN & CLANAC & NURWOM02 & -114.2 & -125.5 & -71.2 \\
\hline PEZQAN & CLANAC11 & NURWOM01 & -114.2 & -130.1 & -67.9 \\
\hline PEZQAN & CLANAC11 & NURWOM02 & -114.2 & -130.1 & -71.2 \\
\hline PIRNOV01 & EHOWIHO1 & IKAYEZ99 & -100.9 & -112.0 & -84.9 \\
\hline PIRNOV01 & EHOWIHO2 & IKAYEZ99 & -100.9 & -112.8 & -84.9 \\
\hline PIRPEN & EHOWIH01 & WEBNAU99 & -103.1 & -112.0 & -87.6 \\
\hline PIRPEN & EHOWIHO2 & WEBNAU99 & -103.1 & -112.8 & -87.6 \\
\hline PIRPIR & EHOWIHO1 & WEBNOI & -106.5 & -112.0 & -83.0 \\
\hline PIRPIR & EHOWIHO2 & WEBNOI & -106.5 & -112.8 & -83.0 \\
\hline PIRQEO & EHOWIH01 & DUTREQ99 & -99.3 & -112.0 & -87.5 \\
\hline PIRQEO & EHOWIHO2 & DUTREQ99 & -99.3 & -112.8 & -87.5 \\
\hline PIRQIS & EHOWIHO1 & WEBNUO & -100.2 & -112.0 & -79.4 \\
\hline PIRQIS & EHOWIHO1 & WEBNUO01 & -100.2 & -112.0 & -79.5 \\
\hline PIRQIS & EHOWIH02 & WEBNUO & -100.2 & -112.8 & -79.4 \\
\hline PIRQIS & EHOWIHO2 & WEBNUO01 & -100.2 & -112.8 & -79.5 \\
\hline PIRREP & EHOWIH01 & PIDGOZ01 & -98.8 & -112.0 & -76.4 \\
\hline PIRREP & EHOWIHO2 & PIDGOZ01 & -98.8 & -112.8 & -76.4 \\
\hline PUJNIS & BENZAC99 & AMCLPY12 & -97.3 & -84.8 & -106.7 \\
\hline PYOCHP & PYRID011 & CHPYRD & -92.9 & -93.3 & -103.6 \\
\hline QAMQUR & PYRAZI & HYQUIN05 & -96.8 & -59.5 & -106.2 \\
\hline QAMQUR & PYRAZIO1 & HYQUIN05 & -96.8 & -63.6 & -106.2 \\
\hline QELYAI & WUHPUK & NOCQED & -99.7 & -92.6 & -109.4 \\
\hline QELYAI & WUHPUK01 & NOCQED & -99.7 & -91.2 & -109.4 \\
\hline QOQPOD & PYRDNO13 & CHORLH01 & -100.5 & -76.0 & -119.8 \\
\hline
\end{tabular}




\begin{tabular}{|c|c|c|c|c|c|}
\hline co-crystal & Co-former 1 & Co-former 2 & Et(PIX) & $\operatorname{Et}(\mathrm{PIX})_{1}$ & $\mathrm{Et}(\mathrm{PIX})_{2}$ \\
\hline QOQPOD & PYRDNO14 & CHORLH01 & -100.5 & -81.1 & -119.8 \\
\hline QOQPOD & PYRDNO15 & CHORLH01 & -100.5 & -80.8 & -119.8 \\
\hline QOQPOD & PYRDNO16 & CHORLH01 & -100.5 & -79.4 & -119.8 \\
\hline QUWZEO & PYRAZI & GUFMAV & -92.6 & -59.5 & -100.7 \\
\hline QUWZEO & PYRAZI01 & GUFMAV & -92.6 & -63.6 & -100.7 \\
\hline QUWZIS & GUFMAV & HEYJOKO1 & -99.6 & -100.7 & -73.2 \\
\hline REHNAT & HYQUIN05 & TCYNBZO1 & -108.0 & -106.2 & -106.7 \\
\hline REQWAM & BDTOLE11 & TCLOBQ & -96.1 & -83.1 & -99.0 \\
\hline REQWAM & BDTOLE11 & TCLOBQ02 & -96.1 & -83.1 & -99.2 \\
\hline RIDFAN & EHOWIHO1 & MNPHOL26 & -107.5 & -112.0 & -98.7 \\
\hline RIDFAN & EHOWIHO2 & MNPHOL26 & -107.5 & -112.8 & -98.7 \\
\hline RIFQAY03 & BDTOLE11 & TCYETY02 & -67.6 & -83.1 & -83.8 \\
\hline RIFQAY03 & BDTOLE11 & TCYETY03 & -67.6 & -83.1 & -74.6 \\
\hline RIJNEF & BDTOLE11 & BAWHEM01 & -90.8 & -83.1 & -86.8 \\
\hline ROKQEN & RAJGUG & MCBZACO2 & -87.5 & -79.0 & -102.9 \\
\hline RUXNAA & IDUWEJ & KIDPIX & -73.3 & -72.1 & -68.8 \\
\hline SAYNEM & SAYNAI & CLACET07 & -114.9 & -146.8 & -85.0 \\
\hline SAYNEM & SAYNAI01 & CLACET07 & -114.9 & -139.7 & -85.0 \\
\hline SENJOK & HYQUIN05 & CUKCIU & -84.6 & -106.2 & -54.6 \\
\hline SENJOK & HYQUIN05 & CUKCIU01 & -84.6 & -106.2 & -55.7 \\
\hline SIDJIA & UREAXX09 & ITIZUG & -95.7 & -104.2 & -64.7 \\
\hline SIDJIA & UREAXX25 & ITIZUG & -95.7 & -105.6 & -64.7 \\
\hline SIVBAA & BNZQUI03 & BDTOLE11 & -73.7 & -60.9 & -83.1 \\
\hline SLCADC10 & UREAXX09 & SALIAC01 & -96.4 & -104.2 & -86.2 \\
\hline SLCADC10 & UREAXX09 & SALIAC15 & -96.4 & -104.2 & -94.1 \\
\hline SLCADC10 & UREAXX25 & SALIAC01 & -96.4 & -105.6 & -86.2 \\
\hline SLCADC10 & UREAXX25 & SALIAC15 & -96.4 & -105.6 & -94.1 \\
\hline SOBNEE & JOZZIH99 & FURACL03 & -107.3 & -122.6 & -115.5 \\
\hline SOLFAC & AMBNACO4 & CYAPYR & -99.0 & -117.0 & -74.0 \\
\hline SOLFAC & AMBNACO6 & CYAPYR & -99.0 & -104.4 & -74.0 \\
\hline SOLFAC & AMBNACO9 & CYAPYR & -99.0 & -106.5 & -74.0 \\
\hline SOLFEG & JOZZIH99 & CYAPYR & -99.3 & -122.6 & -74.0 \\
\hline SUVJEY & AMPYRM10 & TEPHTH12 & -120.3 & -94.5 & -130.0 \\
\hline SUVJEY & AMPYRM10 & TEPHTH13 & -120.3 & -94.5 & -133.6 \\
\hline SUVZEO & NTPYRO11 & BIDLOP02 & -99.3 & -83.2 & -115.4 \\
\hline SUVZEO & NTPYRO11 & BIDLOP99 & -99.3 & -83.2 & -119.7 \\
\hline TETXUL & GIVXUE & CLACET07 & -99.3 & -104.4 & -85.0 \\
\hline TTFCAN14 & BDTOLE11 & TCBENQ07 & -100.3 & -83.1 & -101.7 \\
\hline TTFFAN & BDTOLE11 & TFBENQ & -70.6 & -83.1 & -54.2 \\
\hline TUPRBN01 & PARBAC02 & THIOURO4 & -116.8 & -80.3 & -123.6 \\
\hline TUPRBN01 & PARBACO2 & THIOUR10 & -116.8 & -80.3 & -129.8 \\
\hline UHACIQ & SUNKAO01 & TARTAC01 & -131.0 & -94.0 & -167.6 \\
\hline UHACIQ & SUNKAO01 & TARTAL04 & -131.0 & -94.0 & -165.2 \\
\hline UHACIQ & SUNKAO01 & TARTAM & -131.0 & -94.0 & -151.9 \\
\hline UHACIQ & SUNKAO01 & ZZZDUI01 & -131.0 & -94.0 & -166.3 \\
\hline UHADUD & GLURAC13 & SUNKAO01 & -108.2 & -128.6 & -94.0 \\
\hline UHAFEP & SUNKAO01 & TARTAC01 & -130.1 & -94.0 & -167.6 \\
\hline UHAFEP & SUNKAO01 & TARTAL04 & -130.1 & -94.0 & -165.2 \\
\hline UHAFEP & SUNKAO01 & TARTAM & -130.1 & -94.0 & -151.9 \\
\hline UHAFEP & SUNKAO01 & ZZZDUI01 & -130.1 & -94.0 & -166.3 \\
\hline UMIQIR & CLANAC & WIZFEQ & -106.4 & -125.5 & -71.2 \\
\hline UMIQIR & CLANAC & WIZFEQ01 & -106.4 & -125.5 & -66.7 \\
\hline UMIQIR & CLANAC11 & WIZFEQ & -106.4 & -130.1 & -71.2 \\
\hline UMIQIR & CLANAC11 & WIZFEQ01 & -106.4 & -130.1 & -66.7 \\
\hline USOBOU & CYAPYR & PTOLIC01 & -85.9 & -74.0 & -90.5 \\
\hline VIFKUR & MELAMI03 & URACIL & -138.9 & -150.2 & -114.1 \\
\hline VORCOV & FIXPUX & SUCACB06 & -128.3 & -136.9 & -117.7 \\
\hline WABGEL & BDTOLE11 & TCYNBZ01 & -99.7 & -83.1 & -106.7 \\
\hline WEDKUN & GLURAC13 & AMPYRZ & -118.6 & -128.6 & -99.9 \\
\hline XAPMIK & GEPNOG & OXALACO3 & -117.8 & -96.6 & -102.8 \\
\hline XAPMIK & GEPNOG & OXALACO7 & -117.8 & -96.6 & -107.2 \\
\hline XEBFEQ & BOQQUT02 & ACETAC01 & -80.1 & -88.6 & -63.4 \\
\hline XONPUN & BENZAC99 & XONQAU & -98.7 & -84.8 & -103.9 \\
\hline XONQIC & MCBZAC02 & XONQAU & -104.4 & -102.9 & -103.9 \\
\hline ZAJHOH & AMPYRM10 & PHTHAC01 & -109.0 & -94.5 & -144.3 \\
\hline ZAJJEZ & AMPYRM10 & AMBNZA99 & -107.1 & -94.5 & -117.8 \\
\hline ZODWIY & UREAXX09 & GLURAC13 & -120.3 & -104.2 & -128.6 \\
\hline ZODWIY & UREAXX25 & GLURAC13 & -120.3 & -105.6 & -128.6 \\
\hline co-crystal & Co-former 1 & Co-former 2 & Et(PIX) & $\mathrm{Et}(\mathrm{PIX})_{1}$ & $\mathrm{Et}(\mathrm{PIX})_{2}$ \\
\hline ZOHVUP & UZOXEN & CLANIC06 & -111.6 & -101.4 & -100.7 \\
\hline ZOHWEA & UZOXEN & WEMDUN & -111.7 & -101.4 & -103.9 \\
\hline ZOHWIE & UZOXEN & WEMDOH & -120.0 & -101.4 & -100.7 \\
\hline
\end{tabular}




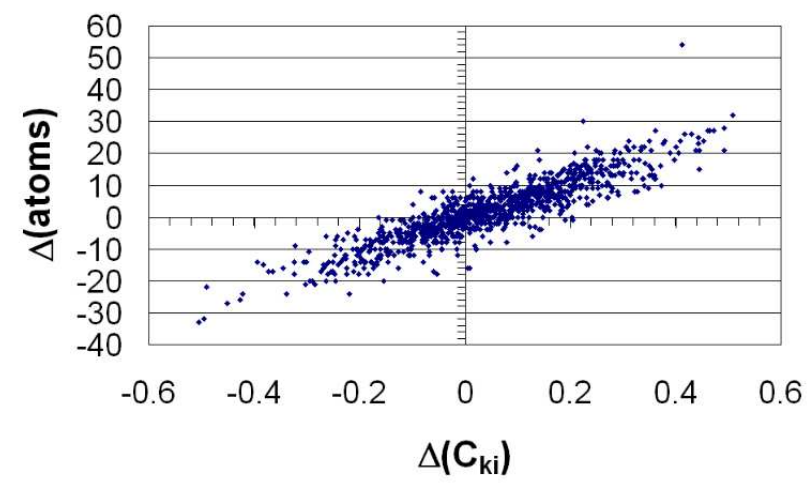

Figure S1. Atom count difference between Co-formers vs. difference of the corresponding partial packing coefficients, $\Delta C_{\mathrm{ki}}$, for structures with 1:1 stoichiometry in the DIM dataset.

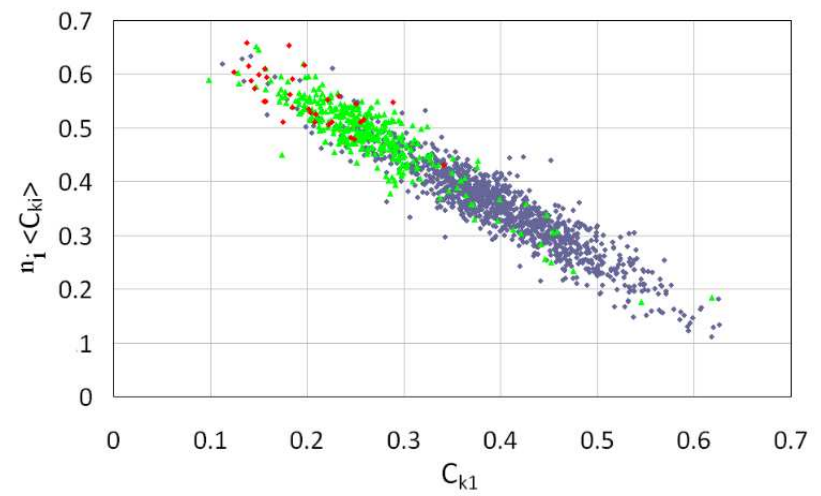

Figure S2. As Figure 1 in the main text, including also also crystals with 1:2 (green points) and 2:2 (red points) stiocihiometries. The latter were brought to the same scale as the 1:1 ones.

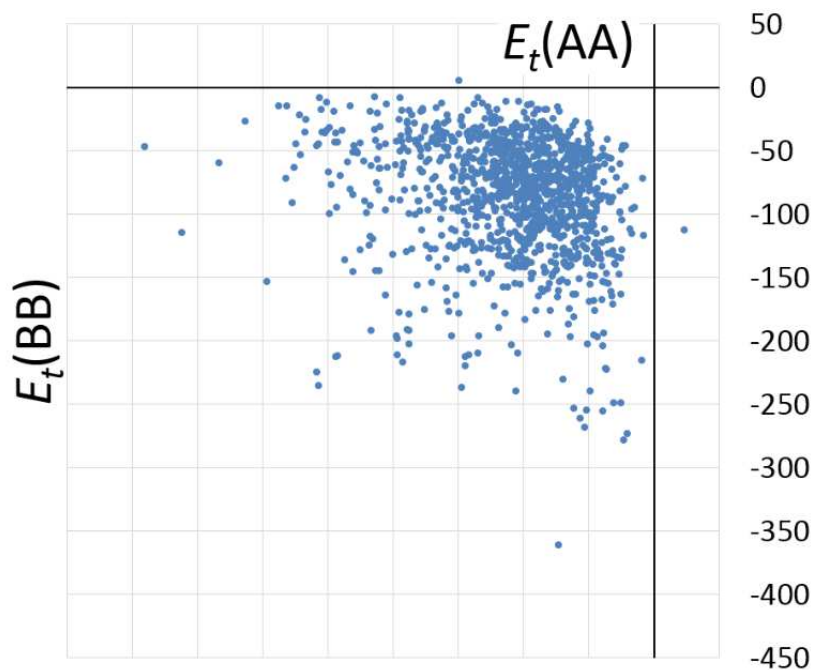

Figure S3. Unbalance in sublattice energies of 1:1 co-crystals. All quantities are given in $\mathrm{kJ} \cdot \mathrm{mol}^{-1}$. 


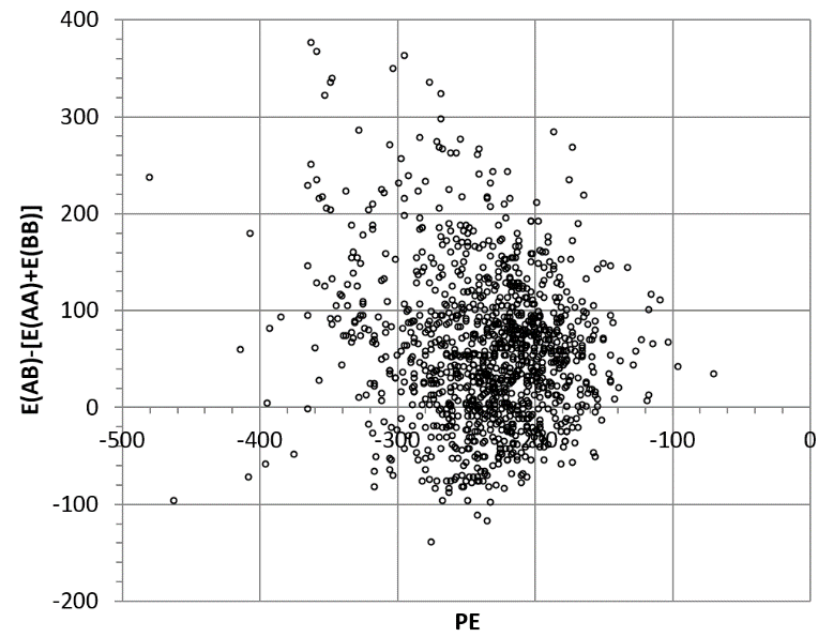

Figure S4. No evident correlations are found between the packing energy PE and the $E_{\text {coup }}$ parameter (see text). Both the quantities are given in $\mathrm{kJ} \cdot \mathrm{mol}^{-1}$.

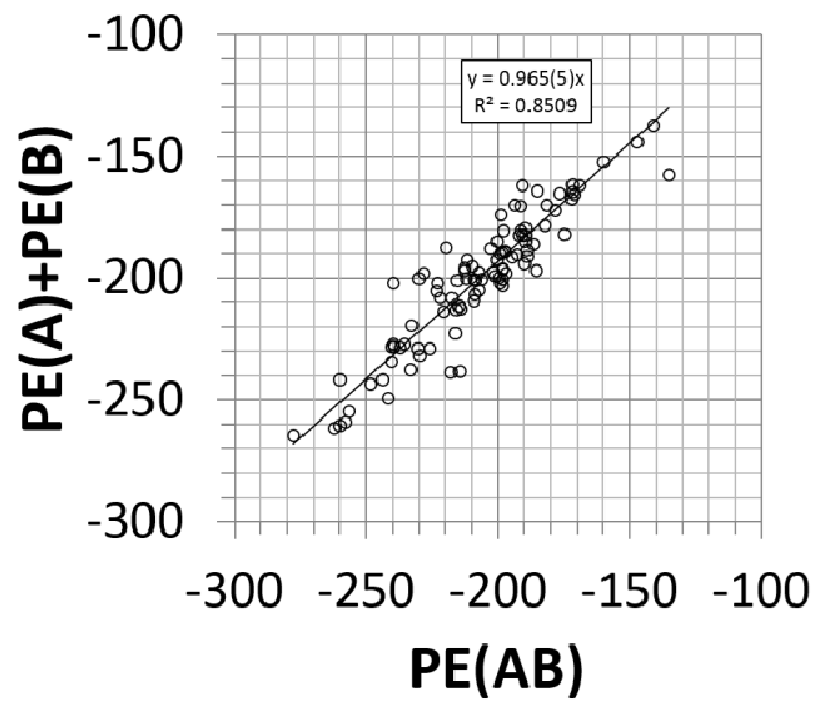

Figure S5. $\mathrm{PE}(\mathrm{AB})$ vs. the sum of $\mathrm{PE}(\mathrm{A})$ and $\mathrm{PE}(\mathrm{B})$. The linear least-squares equation of the straight line is also given, with the estimated error of the angular coefficient. If the intercept is not constrained to 0 , the equation becomes $\mathrm{PE}(\mathrm{AB})=0.941(4) \cdot[\mathrm{PE}(\mathrm{A})+\mathrm{PE}(\mathrm{B})]-5(8)$ with $\mathrm{R}^{2}=0.8514$. All values are given in $\mathrm{kJ} \cdot \mathrm{mol}^{-1}$. 


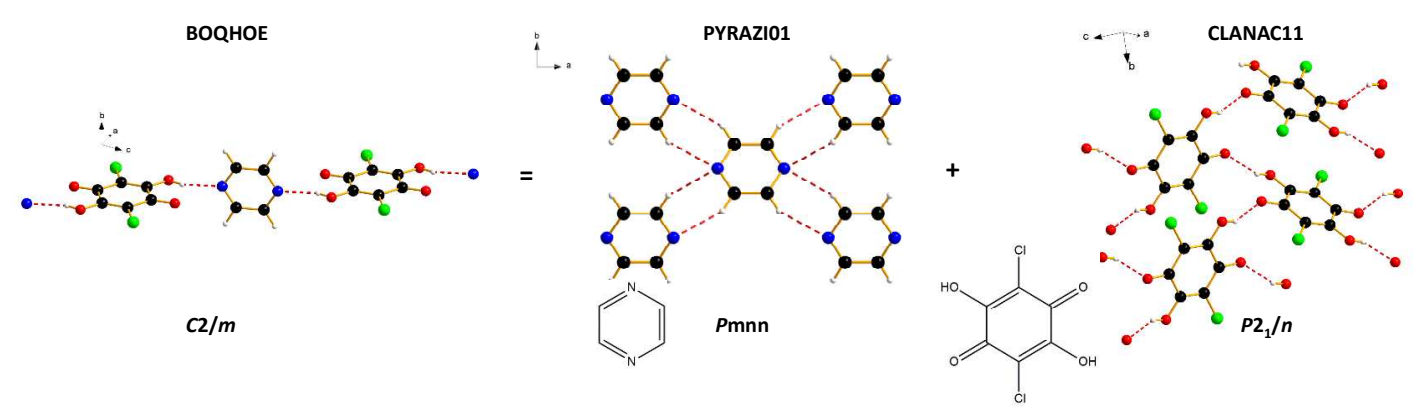

Figure S6. As Fig. 9 in the main text, for the triplet BOQHOE = PYRAZI + CLANAC11.

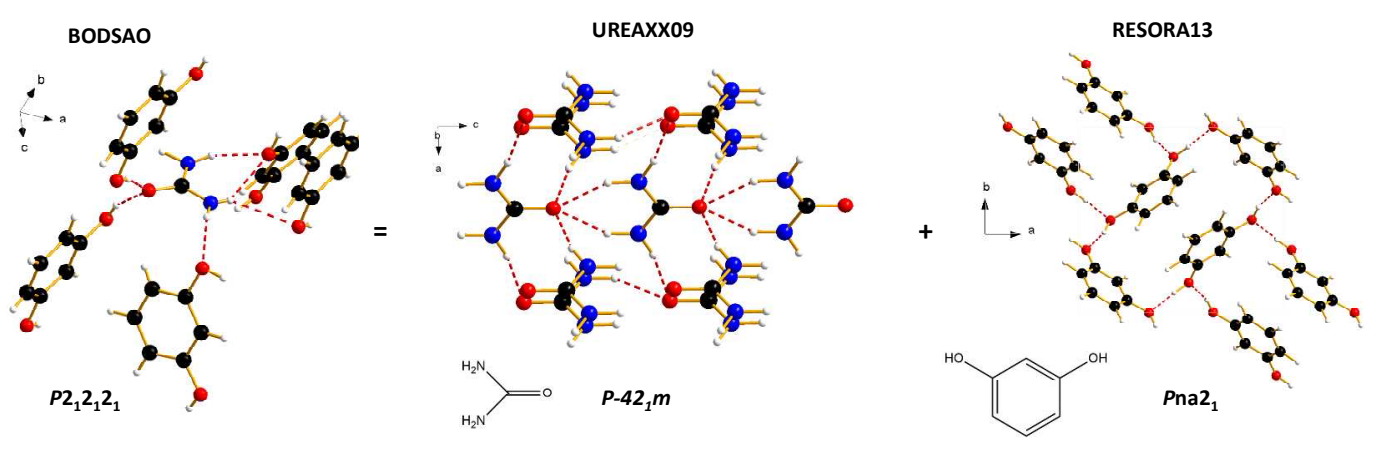

Figure S7. As Fig. 9 in the main text, for the triplet BODSAO = RESORA13 + UREAXX09.

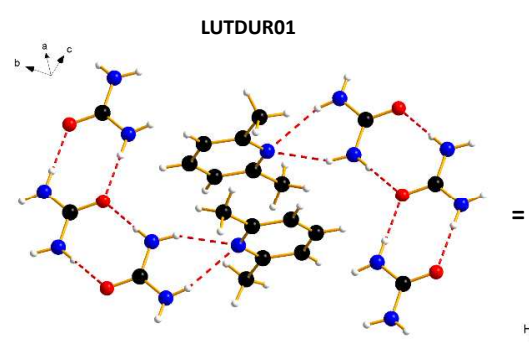

$P-1$

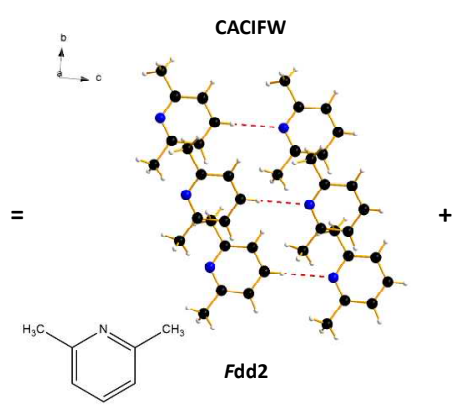

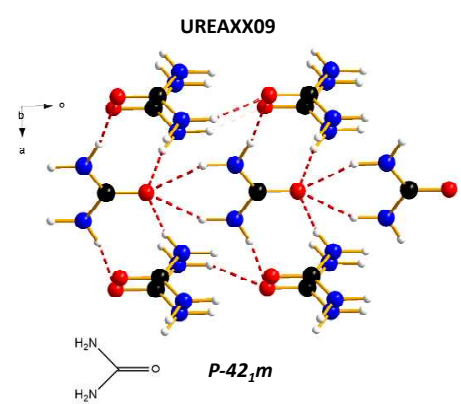

Figure S8. As Fig. 9 in the main text, for the triplet LUTDUR01 = UREAXX09 + CACFIW. 


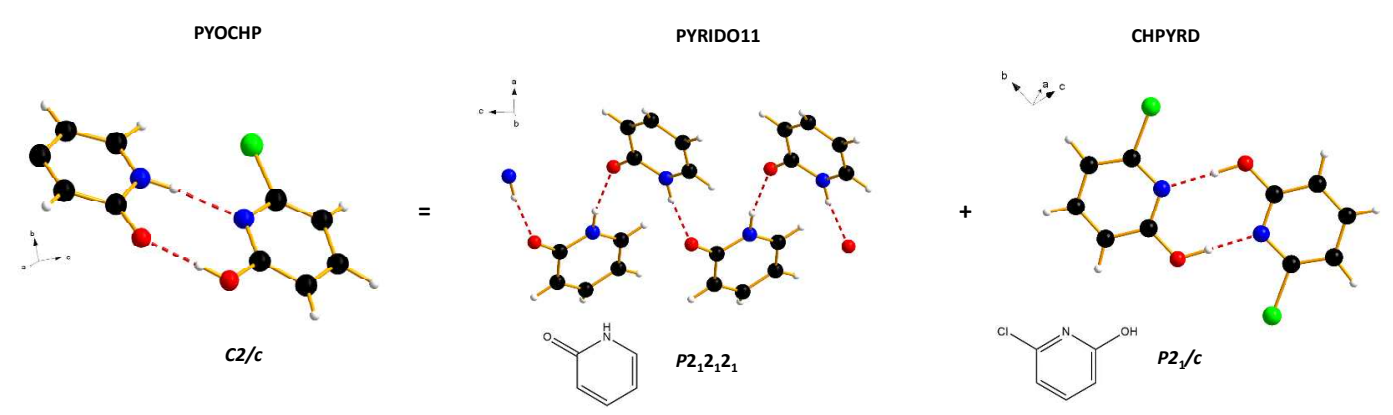

Figure S9. As Fig. 11 in the main text, for the triplet PYOCHP = PYRIDO11 + CHPYRD.

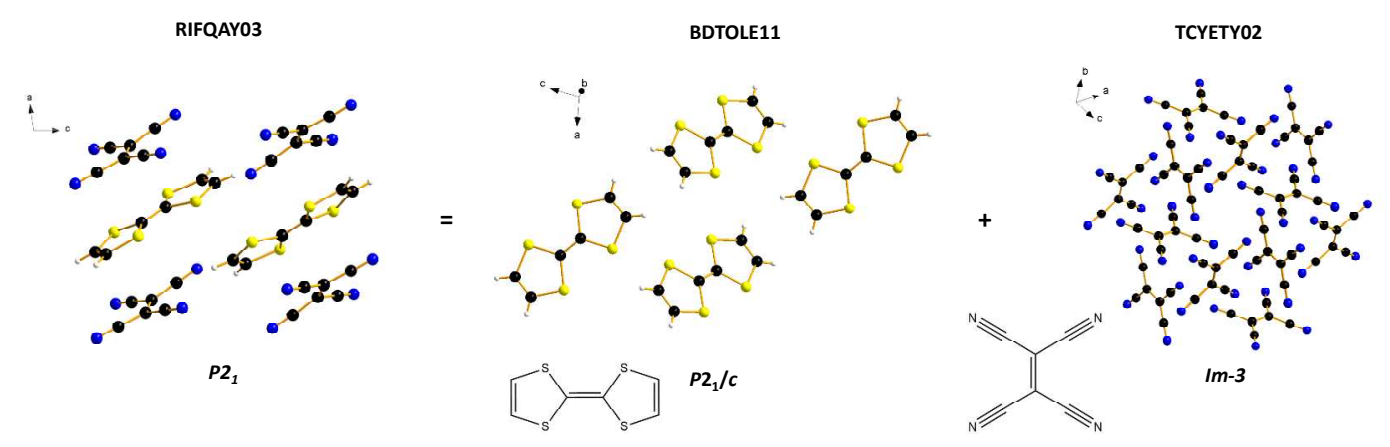

Figure S10. As Fig. 11 in the main text, for the triplet RIFQAY03 = BDTOLE11 + TCYETY02. 


\section{Supplementary information to table 3}

- $\mathbf{A A , A B}$ and BB possible:

- $\quad$ AA only formed (2): GIRPON, SEGFUG: B fragment intramolecular H-bond, bifurcating;

- $\quad$ AA, BB only formed (4): EGENIP, VOHKUA, GAUTAM10, ORUXOP

- $\quad$ none formed (4): LABJIH, PIBDAF, UNUQOK, XUGPEU, intramolecular $\mathrm{NH}^{\cdots \cdots} \mathrm{O}$, weak $\mathrm{NH}^{\cdots \cdots} \mathrm{N}$ or $\mathrm{NH}^{\cdots} \mathrm{S} \mathrm{H}^{-}$ bond not recognized as such

\section{- AA and AB possible:}

- $\quad$ AA only formed (6): BEPPAN, KIJTEE; UCOTIQ, ZEVSIC; AMIMAK and LUTDUR01, weak NH“N AB hydrogen bond

- $\quad$ none formed (16): BAPMAH, CBZTNB, GEXMIH, NAYMOP, PTZPMA, URILAJ, KODXUY, PHNSNB12, REDDEJ, ZEZHET: weak N-H“ O AB H-bond not recognized as such (mostly with $\mathrm{NH}_{2}$ groups, uncertain hydrogens); HARJOB, intramolecular $\mathrm{OH}^{\cdots} \mathrm{O}$ bifurcating with AB H-bond; VIFFEV, LETBIX, PDTCNB, PTZTCQ01 very weak $\mathrm{NH}^{\cdots} \mathrm{N}$ (often $\mathrm{N} \equiv \mathrm{C}$ ) H-bond not recognized as such; YOCZUL, OH hydrogen pointing nowhere (probably wrong);

- AA only possible (6):

- $\quad$ AA only formed: HORVOA, TEXPOB10;

- $\quad$ none formed: ANTPIC, PUNYUS, TERSAK, only N-H' ${ }^{\cdots} \mathrm{O}$ intramolecular, picric acid; EDAWAH, $\mathrm{NH}_{2}$ groups with inaccurate $\mathrm{H}$-atoms. 


\section{References}

1 Gavezzotti, A. Acta Cryst. B 2008, 64, 401

2 Gavezzotti, A. CLP User's manual 2016. Manual, executables, and source codes are freely available at http://www.angelogavezzotti.it 